Z. Wojtkowiak

Nagoya Math. J.

Vol. 177 (2005), 117-153

\title{
ON $l$-ADIC ITERATED INTEGRALS, II FUNCTIONAL EQUATIONS AND $l$-ADIC POLYLOGARITHMS
}

\author{
ZDZISŁAW WOJTKOWIAK
}

\begin{abstract}
We continue to study $l$-adic iterated integrals introduced in the first part. We shall show that the $l$-adic iterated integrals satisfy essentially the same functional equations as the classical complex iterated integrals. Next we are studying $l$-adic analogs of classical polylogarithms.
\end{abstract}

\section{§9. Introduction to Part II}

9.1. The classical complex iterated integrals satisfy functional equations (see [W1]). We shall show that $l$-adic iterated integrals satisfy the same functional equations as the classical complex iterated integrals.

First we introduce the following notation which we shall use in this paper. Let $\pi$ (resp. L) be a group (resp. a Lie algebra). We denote by $\left\{\Gamma^{k} \pi\right\}_{k \geq 1}$ (resp. $\left\{\Gamma^{k} \mathrm{~L}\right\}_{k \geq 1}$ ) the lower central series of the group $\pi$ (resp. the Lie algebra $\mathrm{L}$ ).

We set

$$
g r_{\Gamma}^{k} \pi:=\Gamma^{k} \pi / \Gamma^{k+1} \pi \quad\left(\operatorname{resp} . \quad g r_{\Gamma}^{k} \mathrm{~L}:=\Gamma^{k} \mathrm{~L} / \Gamma^{k+1} \mathrm{~L}\right) .
$$

Before we formulate our main result we make a following remark. Let $Y=\mathbf{P}_{K}^{1} \backslash\left\{b_{1}, \ldots, b_{m+1}\right\}$. Then

$$
\bigoplus_{k=1}^{\infty} g r_{\Gamma}^{k} \pi_{1}(Y(\mathbf{C}) ; x) \otimes \mathbf{Q}
$$

is canonically isomorphic to a free Lie algebra over $\mathbf{Q}$ on $m$ generators $Y_{1}, \ldots, Y_{m}$, which we denote by $\operatorname{Lie}\left(Y_{1}, \ldots, Y_{m}\right)$. Hence any linear form $\varphi$ on $g r_{\Gamma}^{k} \pi_{1}(Y(\mathbf{C}) ; x) \otimes \mathbf{Q}$ corresponds to a linear form $\varphi$ on $\operatorname{Lie}\left(Y_{1}, \ldots, Y_{m}\right)$. Now we formulate our main result concerning functional equations.

Received January 7, 2003.

2000 Mathematics Subject Classification: 11G55, 11G99, $14 \mathrm{G} 32$. 
Theorem D. Let $X=\mathbf{P}_{K}^{1} \backslash\left\{a_{1}, \ldots, a_{n+1}\right\}$ and let $Y=\mathbf{P}_{K}^{1} \backslash\left\{b_{1}, \ldots\right.$, $\left.b_{m+1}\right\}$. Let $z, v \in \hat{X}(K)$. Let $f_{i}: X \rightarrow Y$ be a smooth morphism and let $\varphi_{i} \in \operatorname{Lie}\left(Y_{1}, \ldots, Y_{m}\right)^{\diamond}$ be a linear form of degree $q$ defined over $\mathbf{Q}$ for $i=1, \ldots, N$. Let $n_{1}, \ldots, n_{N}$ be rational numbers. If

$$
\sum_{i=1}^{N} n_{i} \varphi_{i} \circ\left(f_{i}\right)_{*}=0
$$

in $\operatorname{Hom}\left(g r_{\Gamma}^{q} \pi_{1}(X(\mathbf{C}) ; v) ; \mathbf{Q}\right)$, where

$$
\left(f_{i}\right)_{*}: g r_{\Gamma}^{q} \pi_{1}(X(\mathbf{C}) ; v) \longrightarrow g r_{\Gamma}^{q} \pi_{1}\left(Y(\mathbf{C}) ; f_{i}(v)\right)
$$

is the map induced by $f_{i}$ on fundamental groups for $i=1, \ldots, N$, then we have a functional equation

$$
\sum_{i=1}^{N} n_{i} \mathcal{L}^{\varphi_{i}}\left(f_{i}(z), f_{i}(v)\right)=0 .
$$

Next we generalize well known formulas

$$
\int_{a}^{b} \omega+\int_{b}^{a} \omega=0 \text { and } \int_{a}^{c} \omega=\int_{a}^{b} \omega+\int_{b}^{c} \omega
$$

from the elementary calculus ( $\omega$ is a one-form). We show the following result.

Theorem E. Let $z, y, v \in \hat{X}(K)$ and let $\varphi \in \operatorname{Lie}\left(X_{1}, \ldots, X_{n}\right)^{\diamond}$. Then we have

$$
\mathcal{L}^{\varphi}(z, v)+\mathcal{L}^{\varphi}(v, z)=0
$$

and

$$
\mathcal{L}^{\varphi}(z, v)=\mathcal{L}^{\varphi}(z, y)+\mathcal{L}^{\varphi}(y, v) .
$$

Let $\omega_{1}, \omega_{2}$ be one-forms. The classical complex iterated integrals satisfy the following relations written here for two one-forms (see $[\mathrm{Ch}]$ ).

i) $\int_{\gamma} \omega_{1}, \omega_{2}+\int_{\gamma} \omega_{2}, \omega_{1}=\int_{\gamma} \omega_{1} \cdot \int_{\gamma} \omega_{2}$

ii) $\int_{\alpha \beta} \omega_{1}, \omega_{2}=\int_{\alpha} \omega_{1}, \omega_{2}+\int_{\alpha} \omega_{1} \cdot \int_{\beta} \omega_{2}+\int_{\beta} \omega_{1}, \omega_{2}$,

iii) $\int_{\gamma} \omega_{1}, \omega_{2}=(-1)^{2} \int_{\gamma^{-1}} \omega_{2}, \omega_{1}$. 
The analog of the formula i) is satisfied by " $l$-adic iterated integrals" (coefficients of the power series $\Lambda_{p}(\sigma)$ ) by the very definition because the image of the inclusion map of the fundamental group into the algebra of formal non-commutative power series is of the form $\exp L(\mathbf{X})$, where $L(\mathbf{X})$ is the set of Lie elements in the algebra of formal non-commutative power series.

The formula

$$
\mathfrak{f}_{p q}(\sigma)=q^{-1} \cdot \mathfrak{f}_{p}(\sigma) \cdot q \cdot \mathfrak{f}_{q}(\sigma)
$$

(see Part I Lemma 1.0.6), which after using suitable embeddings implies

$$
\Lambda_{p q}(\sigma)=\Lambda_{p}(\sigma) \cdot \Lambda_{q}(\sigma)
$$

is the analog of the formula ii).

We do not know how to show an analog of the formula iii) for " $l$-adic iterated integrals" (coefficients of the power series $\Lambda_{p}(\sigma)$ ). To complete the picture we are still missing several $l$-adic analogs in the following table.

\begin{tabular}{|l|l|}
\hline classical iterated integrals & $l$-adic iterated integrals \\
\hline $\begin{array}{l}\text { values of Riemann zeta function at } \\
\text { positive integers }\end{array}$ & Soulé classes for $\mathbf{Q}$ \\
\hline multivalue zeta numbers & $\begin{array}{l}\text { values of } l \text {-adic iterated integrals } \\
\text { at } 1 \text { and at roots of } 1\end{array}$ \\
\hline multivalue zeta functions & $?$ \\
\hline $\begin{array}{l}\text { shuffle relations for multivalue zeta } \\
\text { numbers and multivalue zeta func- } \\
\text { tions }\end{array}$ & $?$ \\
\hline
\end{tabular}

The classical polylogarithms are the most important examples of iterated integrals. In Section 11 we introduce $l$-adic polylogarithms and we study their properties. We prove a theorem saying when a linear combination of $l$-adic polylogarithms is a cocycle. The reader can compare our result with Proposition in Section 4.6 of [BD]. In Section 11 we study functional equations of $l$-adic polylogarithms. We show that the $l$-adic dilogarithm satisfies the distribution relation

$$
m\left(\sum_{i=0}^{m-1} l_{2}\left(\xi_{m}^{i} z\right)\right)=l_{2}\left(z^{m}\right)
$$

on the Galois group $G_{\mathbf{Q}\left(\mu_{m}\right)}$ and the Abel five term functional equation on $G_{\mathbf{Q}\left(\mu_{l} \infty\right)}$. 
These results are stronger than those in Theorem D in the sense that we get functional equations on the Galois groups $G_{\mathbf{Q}\left(\mu_{n}\right)}$ and $G_{\mathbf{Q}\left(\mu_{l} \infty\right)}$, while the functional equations from Theorem D hold on the subgroup $\bigcap_{i=1}^{N} H_{q}\left(Y ; f_{i}(z), f_{i}(v)\right)$ of $G_{K}$.

\section{$\S 10$. Functional equations}

10.0. Let $X=\mathbf{P}_{K}^{1} \backslash\left\{a_{1}, \ldots, a_{n+1}\right\}$ and let $Y=\mathbf{P}_{K}^{1} \backslash\left\{b_{1}, \ldots, b_{m+1}\right\}$. Let $f: X \rightarrow Y$ be a smooth morphism. Let $z, v \in \hat{X}(K)$. The morphism $f$ induces

$$
f_{*}: \pi_{1}\left(X_{\bar{K}} ; v\right) \longrightarrow \pi_{1}\left(Y_{\bar{K}} ; f(v)\right)
$$

and

$$
f_{*}: \pi\left(X_{\bar{K}} ; z, v\right) \longrightarrow \pi\left(Y_{\bar{K}} ; f(z), f(v)\right) .
$$

Let us fix a path $p$ from $v$ to $z$. We recall that for $\sigma \in G_{K}$ we have defined

$$
\mathfrak{f}_{p}(\sigma):=p^{-1} \cdot \sigma(p)
$$

Then we have

$$
f_{*}\left(\mathfrak{f}_{p}(\sigma)\right)=\mathfrak{f}_{f(p)}(\sigma) .
$$

Let $x=\left(x_{1}, \ldots, x_{n+1}\right)$ (resp. $\left.y=\left(y_{1}, \ldots, y_{m+1}\right)\right)$ be a sequence of geometric generators of $\pi_{1}(X(\mathbf{C}) ; v)$ (resp. $\pi_{1}(Y(\mathbf{C}) ; f(v)$ ). We set $\mathbf{X}:=$ $\left\{X_{1}, \ldots, X_{n}\right\}$ and $\mathbf{Y}:=\left\{Y_{1}, \ldots, Y_{n}\right\}$. We recall that we have embeddings $k_{x}: \pi_{1}(X(\mathbf{C}) ; v) \rightarrow \mathbf{Q}_{l}\{\{\mathbf{X}\}\}$ and $k_{y}: \pi_{1}(Y(\mathbf{C}) ; f(v)) \rightarrow \mathbf{Q}_{l}\{\{\mathbf{Y}\}\}$ associated with a choice of sequences of geometric generators $x$ of $\pi_{1}(X(\mathbf{C}) ; v)$ and $y$ of $\pi_{1}(Y(\mathbf{C}) ; f(v))$. There is a homomorphism of $\mathbf{Q}_{l}$-algebras

$$
f_{\diamond}: \mathbf{Q}_{l}\{\{\mathbf{X}\}\} \longrightarrow \mathbf{Q}_{l}\{\{\mathbf{Y}\}\}
$$

such that

$$
f_{\diamond} \circ k_{x}=k_{y} \circ f_{*} \quad \text { and } \quad f_{\diamond} \circ k_{x, p}=k_{y, f(p)} \circ f_{*}
$$

Let $\sigma \in G_{K\left(\mu_{l} \infty\right)}$. The equations (10.0.1) and (10.0.2) imply that

$$
f_{\diamond} \circ \sigma_{x, p}=\sigma_{y, f(p)} \circ f_{\diamond}
$$

Hence we have

$$
f_{\diamond} \circ \log \sigma_{x, p}=\log \sigma_{y, f(p)} \circ f_{\diamond}
$$


and

$$
f_{\diamond}\left(\left(\log \sigma_{x, p}\right)(1)\right)=\left(\log \sigma_{y, f(p)}\right)(1)
$$

The map $f_{\diamond}$ induces a homomorphism of Lie algebras

$$
f_{\diamond}: L(\mathbf{X}) \longrightarrow L(\mathbf{Y})
$$

Let

$$
f_{\bullet}: \bigoplus_{i=1}^{\infty} g r_{\Gamma}^{i} L(\mathbf{X}) \longrightarrow \bigoplus_{i=1}^{\infty} g r_{\Gamma}^{i} L(\mathbf{Y})
$$

be the map induced by $f_{\diamond}$ on associated graded Lie algebras. The associated graded Lie algebras are canonically isomorphic to $\operatorname{Lie}(\mathbf{X})$ and $\operatorname{Lie}(\mathbf{Y})$. Hence the map $f_{\diamond}$ induces

$$
f_{\bullet}: \operatorname{Lie}(\mathbf{X}) \longrightarrow \operatorname{Lie}(\mathbf{Y})
$$

Let $\varphi \in \operatorname{Lie}(\mathbf{Y})^{\diamond}$ be a linear form of degree $q$. Let us set

$$
a_{x, p}^{\varphi \circ f_{\diamond}}:=\varphi\left(f_{\diamond}\left(\left(\log \sigma_{x, p}\right)(1)\right)\right) .
$$

(In Part I we defined coefficients $a_{x, p}^{\varphi}$ only for homogenous forms, hence we introduce this new definition.) It follows from (10.0.3) that

$$
a_{x, p}^{\varphi \circ f_{\diamond}}=a_{y, f(p)}^{\varphi} .
$$

The map $f_{\diamond}$ is not homogenous. Therefore we have

$$
a_{x, p}^{\varphi \circ f_{\diamond}}=a_{x, p}^{\varphi \circ f_{\bullet}}+\sum_{\operatorname{deg} \chi<q} a_{x, p}^{\chi} .
$$

It follows from (10.0.4) and (10.0.5) that

$$
\mathcal{L}^{\varphi \circ f_{\bullet}}(z, v)=\mathcal{L}^{\varphi}(f(z), f(v))
$$

on the subgroup $H_{q}(X ; z, v)$ of $G_{K}$.

Below we shall use fundamental groups of $X$ or $Y$ with various base points. Sequences of geometric generators and embeddings into algebras of non-commutative formal power series will be chosen as above.

Let $v$ and $v^{\prime}$ belong to $\hat{X}(K)$. If $x=\left(x_{1}, \ldots, x_{n+1}\right)$ is a sequence of geometric generators of $\pi_{1}(X(\mathbf{C}) ; v)$ and $q$ is a path from $v^{\prime}$ to $v$ then 
$q^{-1} \cdot x \cdot q:=\left(q^{-1} \cdot x_{1} \cdot q, \ldots, q^{-1} \cdot x_{n+1} \cdot q\right)$ is a sequence of geometric generators of $\pi_{1}\left(X(\mathbf{C}) ; v^{\prime}\right)$. Then we have embeddings

$$
k_{x}: \pi_{1}(X(\mathbf{C}) ; v) \longrightarrow \mathbf{Q}_{l}\{\{\mathbf{X}\}\}
$$

given by $k_{x}\left(x_{i}\right)=e^{X_{i}}$ for $i=1, \ldots, n$ and

$$
k_{q^{-1} \cdot x \cdot q}: \pi_{1}\left(X(\mathbf{C}) ; v^{\prime}\right) \longrightarrow \mathbf{Q}_{l}\{\{\mathbf{X}\}\}
$$

given by $k_{q^{-1} \cdot x \cdot q}\left(q^{-1} \cdot x_{i} \cdot q\right)=e^{X_{i}}$ for $i=1, \ldots, n$.

TheOrem 10.0.7. Let $f_{i}: X \rightarrow Y$ be a smooth morphism and let $\varphi_{i} \in$ $L\left(Y_{1}, \ldots, Y_{m}\right)^{\diamond}$ be a linear form of degree $q$ defined over $\mathbf{Q}$ for $i=1, \ldots, N$. Let $z, v \in \hat{X}(K)$. Let $n_{1}, \ldots, n_{N}$ be rational numbers. If

$$
\sum_{i=1}^{N} n_{i} \varphi_{i} \circ\left(f_{i}\right)_{*}=0
$$

in $\operatorname{Hom}\left(g r_{\Gamma}^{q} \pi_{1}(X(\mathbf{C}) ; v) ; \mathbf{Q}\right)$, where

$$
\left(f_{i}\right)_{*}: g r_{\Gamma}^{q} \pi_{1}(X(\mathbf{C}) ; v) \longrightarrow g r_{\Gamma}^{q} \pi_{1}\left(Y(\mathbf{C}) ; f_{i}(v)\right)
$$

is the map induced by $f_{i}$ for $i=1, \ldots, N$, then we have functional equations

$$
\sum_{i=1}^{N} n_{i} \mathcal{L}^{\varphi_{i}}\left(f_{i}(z) ; f_{i}(v)\right)=0
$$

on the subgroup $H_{q}(X ; z, v)$ of $G_{K}$ and

$$
\sum_{i=1}^{N} n_{i} a_{y_{i}, f_{i}(p)}^{\varphi_{i}}=\text { lower degree terms }
$$

on $G_{K}$, where "lower degree terms" means a linear combination of $a_{x, p}^{\chi}$ with degree of $\chi$ strictly smaller than $q$ and $y_{i}$ is a sequence of geometric generators of $\pi_{1}\left(Y(\mathbf{C}) ; f_{i}(v)\right)$ for $i=1, \ldots, N$.

Proof. It follows from (10.0.6) that

$$
\begin{aligned}
\sum_{i=1}^{N} n_{i} \mathcal{L}^{\varphi_{i}}\left(f_{i}(z) ; f_{i}(v)\right) & =\sum_{i=1}^{N} n_{i} \mathcal{L}^{\varphi_{i} \circ\left(f_{i}\right) \bullet}(z, v) \\
& =\mathcal{L}^{\sum_{i=1}^{N} n_{i} \varphi_{i} \circ\left(f_{i}\right)} \bullet(z, v)=0 .
\end{aligned}
$$


It follows from (10.0.4) and (10.0.5) that

$$
\begin{aligned}
\sum_{i=1}^{N} n_{i} a_{y_{i}, f_{i}(p)}^{\varphi_{i}} & =\sum_{i=1}^{N} n_{i} a_{x, p}^{\varphi_{i} \circ\left(f_{i}\right)_{\diamond}}=\sum_{i=1}^{N} n_{i} a_{x, p}^{\varphi_{i} \circ\left(f_{i}\right) \bullet}+\text { lower degree terms } \\
& =a_{x, p}^{\sum_{i=1}^{N} n_{i} \varphi_{i} \circ\left(f_{i}\right) \bullet}+\text { lower degree terms }=\text { lower degree terms. }
\end{aligned}
$$

10.1. Let $p$ be a path from $v$ to $z$. Let $x=\left(x_{1}, \ldots, x_{n+1}\right)$ be a sequence of geometric generators of $\pi_{1}(X(\mathbf{C}) ; v)$. Then $x^{\prime}:=\left(p \cdot x_{1} \cdot p^{-1}, \ldots, p \cdot x_{n+1}\right.$. $\left.p^{-1}\right)$ is a sequence of geometric generators of $\pi_{1}(X(\mathbf{C}) ; z)$. The action of $\sigma_{p^{-1}}$ on $\pi_{1}\left(X_{\bar{K}} ; z\right)$ can be expressed in the following way by the action of $\sigma_{p}$ on $\pi_{1}\left(X_{\bar{K}} ; v\right)$. Let $\omega \in \pi_{1}\left(X_{\bar{K}} ; z\right)$. Then $\sigma_{p^{-1}}(\omega)=p \cdot \sigma\left(p^{-1} \cdot \omega \cdot p\right)$. $\mathfrak{f}_{p}(\sigma)^{-1} \cdot p^{-1}$. This implies that on $\mathbf{Q}_{l}\{\{\mathbf{X}\}\}$ we have

$$
\sigma_{x, p}=L_{\Lambda_{p}(\sigma)} \circ \sigma_{x} \quad \text { and } \quad \sigma_{x^{\prime}, p^{-1}}=R_{\Lambda_{p}(\sigma)^{-1}} \circ \sigma_{x}
$$

LEMMA 10.1.1. Let $D$ be a derivation of the algebra $\mathbf{Q}_{l}\{\{\mathbf{X}\}\}$ and let $\omega \in L(\mathbf{X})$. Then

$$
L_{\omega} \bigcirc D=L_{\zeta}+D \quad \text { and } \quad R_{-\omega} \bigcirc D=R_{-\zeta}+D
$$

for some $\zeta \in L(\mathbf{X})$.

Proof. The lemma follows from the identities

$$
\left[L_{\omega}, D\right]=L_{-D(\omega)}, \quad\left[R_{-\omega}, D\right]=R_{D(\omega)}
$$

and

$$
\left[L_{\omega}, L_{-D(\omega)}\right]=L_{-[\omega, D(\omega)]}, \quad\left[R_{-\omega}, R_{D(\omega)}\right]=R_{[\omega, D(\omega)]}
$$

TheOrem 10.1.2. Let $z, v \in \hat{X}(K)$ and let $p$ be a path from $v$ to $z$. Then we have

$$
\begin{aligned}
\text { i) } & \mathcal{L}^{e}(z, v)+\mathcal{L}^{e}(v, z)=0, \\
\text { ii) } & a_{x, p}^{e}+a_{p x p^{-1}, p^{-1}}^{e}=0 .
\end{aligned}
$$

Proof. It follows from (10.1.0) that

$$
\left(\log \sigma_{x^{\prime}, p^{-1}}\right)(1)=\left(R_{-\log \Lambda_{p}(\sigma)} \bigcirc \log \sigma_{x}\right)(1) .
$$

It follows from Lemma 10.1.1 that

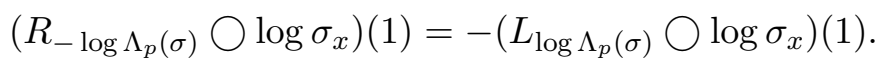


Hence we get that

$$
\left(\log \sigma_{x^{\prime}, p^{-1}}\right)(1)=-\left(\log \sigma_{x, p}\right)(1) .
$$

Evaluating a linear form on both sides of the equation we get the theorem.

TheOREM 10.1.3. Let $z, y, v \in \hat{X}(K)$. Then we have

$$
\mathcal{L}^{e}(z, v)=\mathcal{L}^{e}(z, y)+\mathcal{L}^{e}(y, v) .
$$

Proof. Let $p$ be a path from $v$ to $y$, let $r$ be a path from $y$ to $z$ and let $q=r \cdot p$. We have $\sigma_{p}=L_{\mathfrak{f}_{p}(\sigma)} \circ \sigma$ and $\sigma_{q}=L_{\mathfrak{f}_{q}(\sigma)} \circ \sigma$ on $\pi_{1}\left(X_{\bar{K}} ; v\right)$ and $\sigma_{r}=$ $L_{\mathfrak{f}_{r}(\sigma)} \odot \sigma$ on $\pi_{1}\left(X_{\bar{K}} ; y\right)$. It follows from Lemma 1.0.6 that $\sigma_{q}=L_{p^{-1} \mathfrak{f}_{r}(\sigma) p} \circ \sigma_{p}$. Let us choose a sequence $x$ of geometric generators of $\pi_{1}\left(X_{\bar{K}} ; y\right)$. Then $x^{\prime}=p^{-1} \cdot x \cdot p$ is a sequence of geometric generators of $\pi_{1}\left(X_{\bar{K}} ; v\right)$. Observe that

$$
\sigma_{x^{\prime}, q}=\sigma_{x, r} \circ \sigma_{x}^{-1} \circ \sigma_{x^{\prime}, p}
$$

Hence we get

$$
\log \sigma_{x^{\prime}, q}=\log \sigma_{x, r} \bigcirc \log \sigma_{x}^{-1} \bigcirc \log \sigma_{x^{\prime}, p} .
$$

Let $\sigma$ belongs to the degree $m$ step of the filtration defined in Section 3, i.e., $\sigma \in \mathcal{K}_{m}^{T}(X)$ for some finite subset $T \subset \hat{X}(K)^{2}$. Then

$$
\left(\log \sigma_{x^{\prime}, q}\right)(1) \equiv\left(\log \sigma_{x, r}\right)(1)+\left(\log \sigma_{x^{\prime}, p}\right)(1) \bmod \Gamma^{m+1} L(\mathbf{X})
$$

Evaluating a linear form of degree $m$ on both sides of the congruence we get the theorem.

10.2. It follows from Proposition 7.1.10 that relations between functions $\mathcal{L}^{e}(z, v)$ imply relations between symbols $\{z, v\}_{e}$. Hence we get the following result.

Corollary 10.2.1. Assume that Conjectures $D_{n}$ are true for all $n$. Assume that for all $n$ the maps realization : $\operatorname{Ext}_{\mathcal{M M}_{K}}^{1}(\mathbf{Q}(0), \mathbf{Q}(n)) \otimes \mathbf{Q} \rightarrow$ $H^{1}\left(G_{K}, \mathbf{Q}_{l}(n)\right)$ are injective. Then we have

$$
\{z, v\}_{e}+\{v, z\}_{e}=0
$$

and

$$
\{z, v\}_{e}=\{z, y\}_{e}+\{y, v\}_{e}
$$

in $\mathcal{L}^{K}(X)$.

Proof. The corollary follows from Theorems 10.1.2 and 10.1.3 and Proposition 7.1.10. 
10.3. Let $\pi$ be a group. If $\pi$ is nilpotent then we denote by $\pi \otimes \mathbf{Q}$ its rationalization. For an arbitrary group $\pi, \pi \otimes \mathbf{Q}:=\lim _{n}\left(\left(\pi / \Gamma^{n} \pi\right) \otimes \mathbf{Q}\right)$ is a rational completion of $\pi$. The group $\pi_{1}\left(X_{\bar{K}} ; v\right)$ is equipped with a pro-finite topology. Hence every quotient $\pi_{1}\left(X_{\bar{K}} ; v\right) / \Gamma^{n} \pi_{1}\left(X_{\bar{K}} ; v\right)$ is equipped with a pro-finite topology. Therefore rationalization $\left(\pi_{1}\left(X_{\bar{K}} ; v\right) / \Gamma^{n} \pi_{1}\left(X_{\bar{K}} ; v\right)\right) \otimes \mathbf{Q}$ is a $\mathbf{Q}_{l}$-Lie group. Hence $\pi_{1}\left(X_{\bar{K}} ; v\right) \otimes \mathbf{Q}=\lim _{n}\left(\left(\pi_{1}\left(X_{\bar{K}} ; v\right) / \Gamma^{n} \pi_{1}\left(X_{\bar{K}} ; v\right)\right) \otimes\right.$ $\mathbf{Q})$ is equipped with a topology of the inverse limit of $\mathbf{Q}_{l}$-Lie groups. The action of $G_{K}$ on $\pi_{1}\left(X_{\bar{K}} ; v\right)$ extends uniquely to a continous action of $G_{K}$ on $\pi_{1}\left(X_{\bar{K}} ; v\right) \otimes \mathbf{Q}$.

Now we shall define a rational completion of $\pi_{1}\left(X_{\bar{K}} ; v\right)$-torsor $\pi\left(X_{\bar{K}}\right.$; $z, v)$. We introduce an equivalence relation on the product $\pi\left(X_{\bar{K}} ; z, v\right) \times$ $\pi_{1}\left(X_{\bar{K}} ; v\right) \otimes \mathbf{Q}$. We say that a pair $(p, S)$ is equivalent to a pair $(q, T)$ and we write $(p, S) \sim(q, T)$ if $S=\left(p^{-1} \cdot q\right) \cdot T$ in $\pi_{1}\left(X_{\bar{K}} ; v\right) \otimes \mathbf{Q}$.

We set

$$
\pi\left(X_{\bar{K}} ; z, v\right) \otimes \mathbf{Q}:=\left(\pi\left(X_{\bar{K}} ; z, v\right) \times \pi_{1}\left(X_{\bar{K}} ; v\right) \otimes \mathbf{Q}\right) / \sim .
$$

The Galois group $G_{K}$ acts on the product $\pi\left(X_{\bar{K}} ; z, v\right) \times \pi_{1}\left(X_{\bar{K}} ; v\right) \otimes \mathbf{Q}$ component wise. The group $\pi_{1}\left(X_{\bar{K}} ; v\right) \otimes \mathbf{Q}$ acts on the product $\pi\left(X_{\bar{K}} ; z, v\right) \times$ $\pi_{1}\left(X_{\bar{K}} ; v\right) \otimes \mathbf{Q}$ by the right multiplication on the second factor. Both actions are compatible with the equivalence relation $\sim$ and continous. Hence $G_{K}$ acts on the set of equivalence classes $\pi\left(X_{\bar{K}} ; z, v\right) \otimes \mathbf{Q}$. The action of $\pi_{1}\left(X_{\bar{K}} ; v\right) \otimes \mathbf{Q}$ on the product $\pi\left(X_{\bar{K}} ; z, v\right) \times \pi_{1}\left(X_{\bar{K}} ; v\right) \otimes \mathbf{Q}$ induces a structure of $\pi_{1}\left(X_{\bar{K}} ; v\right) \otimes \mathbf{Q}$-torsor on the set of equivalence classes $\pi\left(X_{\bar{K}} ; z, v\right) \otimes \mathbf{Q}$. Elements of $\pi\left(X_{\bar{K}} ; z, v\right) \otimes \mathbf{Q}$ have the form $p \cdot S$, where $p$ is in $\pi\left(X_{\bar{K}} ; z, v\right)$ and $S \in \pi_{1}\left(X_{\bar{K}} ; v\right) \otimes \mathbf{Q}$. We shall call them $\mathbf{Q}_{l}$-paths.

Lemma 10.3.1. The embedding $k_{x}: \pi_{1}\left(X_{\bar{K}} ; v\right) \rightarrow \mathbf{Q}_{l}\{\{\mathbf{X}\}\}$ extends uniquely to a continous multiplicative embedding $\bar{k}_{x}: \pi_{1}\left(X_{\bar{K}} ; v\right) \otimes \mathbf{Q} \rightarrow$ $\mathbf{Q}_{l}\{\{\mathbf{X}\}\}$.

Proof. The image of $k_{x}$ is contained in $\mathbf{Q}_{l}\{\{\mathbf{X}\}\}^{*}$. The group $\mathbf{Q}_{l}\{\{\mathbf{X}\}\}^{*}$ is a pro-unipotent group with exponents in $\mathbf{Q}_{l}$. Hence $k_{x}$ extends to $\bar{k}_{x}$ : $\pi_{1}\left(X_{\bar{K}} ; v\right) \otimes \mathbf{Q} \rightarrow \mathbf{Q}_{l}\{\{\mathbf{X}\}\}$.

Further we shall denote the embedding $\bar{k}_{x}$ by $k_{x}$. One shows that the formulas

$$
\begin{aligned}
& \mathfrak{f}_{p \cdot q}(\sigma)=q^{-1} \cdot \mathfrak{f}_{p}(\sigma) \cdot q \cdot \mathfrak{f}_{q}(\sigma), \\
& \Lambda_{p \cdot q}(\sigma)=\Lambda_{p}(\sigma) \cdot \Lambda_{q}(\sigma) \\
& \Lambda_{p}(\tau \cdot \sigma)=\Lambda_{p}(\tau) \cdot \tau\left(\Lambda_{p}(\sigma)\right)
\end{aligned}
$$


and $g_{*}\left(\mathfrak{f}_{p}\right)=\mathfrak{f}_{g(p)}$, where $g: X_{K} \rightarrow X_{K}$ is a regular map, are valid also for $\mathrm{Q}_{l}$-paths $p$ and $q$.

\section{$\S 11 . \quad l$-adic polylogarithms}

11.0. In this subsection we introduce $l$-adic polylogarithms. We give sufficient conditions when a linear combination of $l$-adic polylogarithms is a cocycle. Next we are studying a relative version of $l$-adic polylogarithms. We also show that $l$-adic polylogarithms are special case of $l$-adic iterated integrals introduced in Section 5.

Let $K$ be a number field. Let $V=\mathbf{P}_{K}^{1} \backslash\{0,1, \infty\}$. Let $x$ and $y$ be standard generators of $\pi_{1}\left(V_{\bar{K}} ; \overrightarrow{01}\right)$ - loops around 0 and 1 respectively (see the Picture 1).

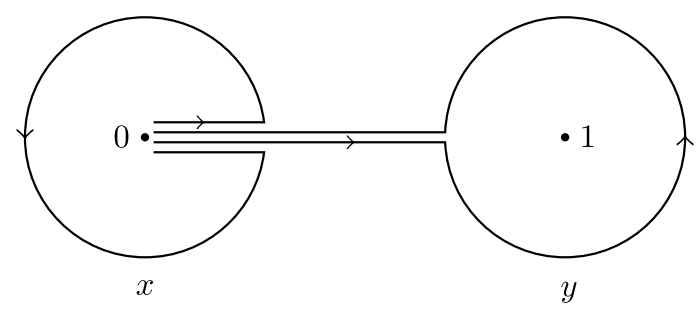

Picture 1

Let $k: \pi_{1}\left(V_{\bar{K}} ; \overrightarrow{01}\right) \otimes \mathbf{Q} \rightarrow \mathbf{Q}_{l}\{\{X, Y\}\}$ be a multiplicative continous embedding given by $k(x)=e^{X}$ and $k(y)=e^{Y}$. We denote by $\operatorname{Lie}(X, Y)$ a free Lie algebra over $\mathbf{Q}_{l}$ on $X$ and $Y$ and by $L(X, Y)$ a completion of $\operatorname{Lie}(X, Y)$ with respect to the lower central series. We identify $L(X, Y)$ with the Lie algebra of Lie elements in $\mathbf{Q}_{l}\{\{X, Y\}\}$.

Let us set $E_{1}:=Y, E_{k+1}:=\left[E_{k}, X\right]$. Let $\mathcal{B}$ be a base of $\operatorname{Lie}(X, Y)$ given by basic Lie elements. We assume that $E_{k} \in \mathcal{B}$ for $k=1,2, \ldots$.

Let $z \in \hat{V}(K)$ and let $p$ be a $\mathbf{Q}_{l}$-path from $\overrightarrow{01}$ to $z$. We recall that $\mathfrak{f}_{p}(\sigma)=p^{-1} \cdot \sigma(p) \in \pi_{1}\left(V_{\bar{K}} ; \overrightarrow{01}\right) \otimes \mathbf{Q}$ and $\Lambda_{p}(\sigma):=k\left(\mathfrak{f}_{p}(\sigma)\right) \in \mathbf{Q}_{l}\{\{X, Y\}\}$ for any $\sigma \in G_{K}$.

If $e \in \mathcal{B}$ we denote by $e^{*}$ the dual linear form to $e$ with respect to $\mathcal{B}$.

Definition 11.0.1. Let $\sigma \in G_{K}$. We set

$$
l_{n}(z)(\sigma):=E_{n}^{*}\left(\log \Lambda_{p}(\sigma)\right) \quad \text { and } \quad l(z)(\sigma):=X^{*}\left(\log \Lambda_{p}(\sigma)\right) .
$$

The coefficient $l_{n}(z)$ is an $l$-adic polylogarithm $(n$-th order $l$-adic poly$\operatorname{logarithm}$ ) evaluated at $z$. It is a function from $G_{K}$ to $\mathbf{Q}_{l}(n)$. It depends on 
a choice of $p$ in $\pi\left(V_{\bar{K}} ; z, \overrightarrow{01}\right) \otimes \mathbf{Q}$. The coefficient $l(z)$ is an $l$-adic logarithm evaluated at $z$. If we are using various paths and it is important to indicate the dependence of $l_{n}(z)$ (resp. $\left.l(z)\right)$ on a path $p$ we shall write $l_{n}(z)_{p}$ (resp. $\left.l(z)_{p}\right)$.

DeFinition 11.0.2. We set

$$
\mathcal{L}_{n}(z):=l_{n}(z)_{\mid H_{n}(V ; z, \overrightarrow{01})} .
$$

Observe that $\mathcal{L}_{n}(z)$ depends only on $z$.

Let us set $e_{1}:=y$ and $e_{k+1}:=\left(e_{k}, x\right)$. Observe that any element $g \in \pi_{1}\left(V_{\bar{K}} ; \overrightarrow{01}\right) \otimes \mathbf{Q}$ can be written in the following form $g=x^{\alpha^{0}(g)} \cdot y^{\alpha^{1}(g)} \cdot e_{2}^{\alpha^{2}(g)} \cdot e_{3}^{\alpha^{3}(g)} \cdot((y, x) y)^{\beta(g)} \cdot e_{4}^{\alpha^{4}(g)} \cdot f_{4} \cdots e_{n}^{\alpha^{n}(g)} \cdot f_{n} \cdots$,

where the exponents are in $\mathbf{Q}_{l}$ and each $f_{n}$ is a product of powers of commutators of length $n$, which contain $y$ at least twice.

Definition 11.0.3. Let $\sigma \in G_{K}$. We define functions $\kappa_{z}^{n}: G_{K} \rightarrow \mathbf{Q}_{l}$ by the identity

$$
\mathfrak{f}_{p}(\sigma)=x^{\kappa_{z}^{0}(\sigma)} \cdot y^{\kappa_{z}^{1}(\sigma)} \cdot e_{2}^{\kappa_{z}^{2}(\sigma)} \cdot e_{3}^{\kappa_{z}^{3}(\sigma)} \cdot f_{3} \cdot e_{4}^{\kappa_{z}^{4}(\sigma)} \cdot f_{4} \cdots e_{n}^{\kappa_{z}^{n}(\sigma)} \cdot f_{n} \cdots
$$

Let $n \geq 1$. Then $\kappa_{z}^{n}$ we view as a function from $G_{K}$ to $\mathbf{Q}_{l}(n) . \kappa_{z}^{0}$ we view as a function from $G_{K}$ to $\mathrm{Q}_{l}(1)$. We shall also use the notation $\kappa_{0}(z):=\kappa_{z}^{0}$ and $\kappa_{1}(z):=\kappa_{z}^{1}$. If we are using various paths and it is important to indicate the dependence of $\kappa_{z}^{n}(\sigma)$ on a path $p$ we shall write $\kappa_{z}^{n}(\sigma)_{p}$

We shall express $l$-adic polylogarithms in terms of functions $\kappa_{z}^{n}$.

Let $f \in L(X, Y)$. We define a derivation ad $f$ of $L(X, Y)$ setting $($ ad $f)(g)=[f, g]$ for any $g \in L(X, Y)$.

Let $I_{k}$ be a Lie ideal of $L(X, Y)$ generated topologically by Lie brackets which contain $Y$ at least $k$-times.

LEMMA 11.0.4. We have

$$
\log \left(k\left(e_{n+1}\right)\right)=(-1)^{n} \sum_{k_{1}, \ldots, k_{n}=1}^{\infty} \frac{1}{k_{1} ! \cdots k_{n} !}(\operatorname{ad} X)^{k_{1}+\cdots+k_{n}}(Y) \bmod I_{2} .
$$


LEMmA 11.0.5. (see [B] chapitre II) We have

$$
\log \left(e^{X} \cdot e^{Y}\right)=X+Y+\frac{1}{2}[X, Y]+\sum_{n=1}^{\infty} \frac{1}{(2 n) !} B_{2 n}(a d X)^{2 n}(Y) \bmod I_{2} .
$$

Proposition 11.0.6. Let $\sigma \in G_{K}$. We have

$$
\begin{aligned}
& \log \Lambda_{p}(\sigma)=\kappa_{z}^{0}(\sigma) X \\
& +\sum_{i=1}^{\infty}(-1)^{i-1} \kappa_{z}^{i}(\sigma)\left(\sum_{k_{1}, \ldots, k_{i-1}=1}^{\infty} \frac{1}{k_{1} ! \cdots k_{i-1} !}(\operatorname{ad} X)^{\left.k_{1}+\cdots+k_{i-1}(Y)\right)}\right. \\
& +\frac{1}{2}\left[\kappa_{z}^{0}(\sigma) X, \sum_{i=1}^{\infty}(-1)^{i-1} \kappa_{z}^{i}(\sigma)\right. \\
& \quad \times\left(\sum_{k_{1}, \ldots, k_{i-1}=1}^{\infty} \frac{1}{k_{1} ! \cdots k_{i-1} !}(a d X)^{\left.k_{1}+\cdots+k_{i-1}(Y)\right)}\right] \\
& +\sum_{n=1}^{\infty} \frac{\left(\kappa_{z}^{0}(\sigma)\right)^{2 n} B_{2 n}(a d X)^{2 n}\left(\sum_{i=1}^{\infty}(-1)^{i-1} \kappa_{z}^{i}(\sigma)\right.}{(2 n) !}\left(\sum_{k_{1}, \ldots, k_{i-1}=1}^{\infty} \frac{1}{k_{1} ! \cdots k_{i-1} !}(a d X)^{\left.k_{1}+\cdots+k_{i-1}(Y)\right)} \bmod I_{2} .\right.
\end{aligned}
$$

Proof. The proposition follows from Lemmas 11.0.4 and 11.0.5.

Using Proposition 11.0.6 we can easily calculate $l$-adic polylogarithms in terms of functions $\kappa_{z}^{n}$. For example in small degrees we get the following result.

Corollary 11.0.7. We have

$$
l(z)=\kappa_{z}^{0}, \quad l_{1}(z)=\kappa_{z}^{1}, \quad l_{2}(z)=\kappa_{z}^{2}-\frac{1}{2} \kappa_{z}^{0} \cdot \kappa_{z}^{1}
$$

and

$$
l_{3}(z)=\kappa_{z}^{3}-\frac{1}{2} \kappa_{z}^{0} \cdot \kappa_{z}^{2}+\frac{1}{12}\left(\kappa_{z}^{0}\right)^{2} \cdot \kappa_{z}^{1}-\frac{1}{2} \kappa_{z}^{2}
$$

Proposition 11.0.8. Let $\zeta \in \hat{V}(K)$ and let $p$ be a $\mathbf{Q}_{l}$-path from $\overrightarrow{01}$ to $\zeta$. Let $q$ be the standard path from $\overrightarrow{01}$ to $\overrightarrow{10}$ (an interval $[0,1])$. Let $g: V_{K} \rightarrow V_{K}$ be given by $g(z)=1-z$. Then we have

$$
l_{1}(\zeta)_{p}=l(1-\zeta)_{g(p) \cdot q} .
$$


Proof. It follows from Corollary 11.0.7 that

$$
\mathfrak{f}_{p} \equiv x^{l(\zeta)_{p}} \cdot y^{l_{1}(\zeta)_{p}} \bmod \Gamma^{2}\left(\pi_{1}\left(V_{\bar{K}} ; \overrightarrow{01}\right) \otimes \mathbf{Q}\right) .
$$

Observe that $g(p) \cdot q$ is a $\mathbf{Q}_{l}$-path from $\overrightarrow{01}$ to $1-\zeta$. Hence we have

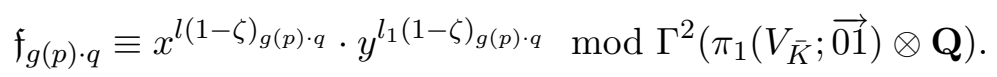

On the other side

$$
\begin{aligned}
\mathfrak{f}_{g(p) \cdot q} & =q^{-1} \cdot \mathfrak{f}_{g(p)} \cdot q \cdot \mathfrak{f}_{q}=q^{-1} \cdot g_{*}\left(\mathfrak{f}_{p}\right) \cdot q \cdot \mathfrak{f}_{q} \\
& \equiv x^{l_{1}(\zeta)_{p}} \cdot y^{l(\zeta)_{p}} \bmod \Gamma^{2}\left(\pi_{1}\left(V_{\bar{K}} ; \overrightarrow{01}\right) \otimes \mathbf{Q}\right)
\end{aligned}
$$

because $q^{-1} \cdot g_{*}(x) \cdot q=y, q^{-1} \cdot g_{*}(y) \cdot q=x$ and $\mathfrak{f}_{q} \equiv 1 \bmod \Gamma^{2}\left(\pi_{1}\left(V_{\bar{K}} ; \overrightarrow{01}\right) \otimes\right.$ $\mathrm{Q})$. The proposition follows from the last two congruences.

Theorem 11.0.9. Let $z_{i} \in \hat{V}(K)$, let $p_{i} \in \pi\left(V_{\overline{\mathbf{Q}}} ; z_{i}, \overrightarrow{01}\right) \otimes \mathbf{Q}$ and let $n_{i} \in \mathbf{Q}_{l}$ for $i=1, \ldots, N$. Let us assume that l-adic polylogarithms $l_{k}\left(z_{i}\right)$ calculated along the $\mathbf{Q}_{l}$-paths $p_{i}$ for $i=1, \ldots, N$ satisfy the following conditions

i) $\sum_{i=1}^{N} n_{i}\left(l\left(z_{i}\right)(\tau)\right)^{\alpha} \cdot\left(l\left(z_{i}\right)(\sigma)\right)^{\beta} \cdot\left(l\left(z_{i}\right)(\tau) \cdot l_{1}\left(z_{i}\right)(\sigma)-l\left(z_{i}\right)(\sigma) \cdot l_{1}\left(z_{i}\right)(\tau)\right)=$ 0 for any $\tau, \sigma \in G_{K}$ and for any $\alpha$ and $\beta$ such that $\alpha+\beta=n-2$,

ii) $\sum_{i=1}^{N} n_{i}\left(l\left(z_{i}\right)(\tau)\right)^{\alpha} \cdot\left(l\left(z_{i}\right)(\sigma)\right)^{\beta} \cdot l_{k}\left(z_{i}\right)(\sigma)=0$ for any $\tau, \sigma \in G_{K}$, for $k=2, \ldots, n-1$ and for any $\alpha$ and $\beta$ such that $\alpha+\beta=n-k$.

Then $\sum_{i=1}^{N} n_{i} l_{n}\left(z_{i}\right)$ is a cocycle on $G_{K}$ with values in $\mathbf{Q}_{l}(n)$.

Proof. The equality $\Lambda_{p}(\tau \sigma)=\Lambda_{p}(\tau) \cdot \tau\left(\Lambda_{p}(\sigma)\right)$ implies

$$
\begin{aligned}
& \log \Lambda_{p}(\tau \sigma)=\log \Lambda_{p}(\tau)+\log \tau\left(\Lambda_{p}(\sigma)\right)+\frac{1}{2}\left[\log \Lambda_{p}(\tau), \log \left(\tau\left(\Lambda_{p}(\sigma)\right)\right)\right] \\
& \quad-\frac{1}{12}\left[\left[\log \Lambda_{p}(\tau), \log \left(\tau\left(\Lambda_{p}(\sigma)\right)\right)\right], \log \Lambda_{p}(\tau)\right] \\
& \quad+\frac{1}{12}\left[\left[\log \Lambda_{p}(\tau), \log \left(\tau\left(\Lambda_{p}(\sigma)\right)\right)\right], \log \left(\tau\left(\Lambda_{p}(\sigma)\right)\right)\right] \\
& \quad-\frac{1}{24}\left[\left[\left[\log \Lambda_{p}(\tau), \log \left(\tau\left(\Lambda_{p}(\sigma)\right)\right)\right], \log \left(\tau\left(\Lambda_{p}(\sigma)\right)\right)\right], \log \Lambda_{p}(\tau)\right]+\cdots
\end{aligned}
$$


Comparing coefficients at $E_{n}$ we get

$$
\begin{aligned}
& l_{n}(z)(\tau \sigma)=l_{n}(z)(\tau)+\chi(\tau)^{n} l_{n}(z)(\sigma) \\
& +\frac{1}{2}\left(l_{n-1}(z)(\tau) \chi(\tau) l(z)(\sigma)-\chi(\tau)^{n-1} l_{n-1}(z)(\sigma) l(z)(\tau)\right) \\
& -\frac{1}{12}\left(l_{n-2}(z)(\tau) \chi(\tau) l(z)(\tau) l(z)(\sigma)-\chi(\tau)^{n-2} l_{n-2}(z)(\sigma)(l(z)(\tau))^{2}\right) \\
& +\frac{1}{12}\left(l_{n-2}(z)(\tau) \chi(\tau)^{2}(l(z)(\sigma))^{2}-\chi(\tau)^{n-1} l_{n-2}(z)(\sigma) l(z)(\tau) l(z)(\sigma)\right) \\
& -\frac{1}{24}\left(l_{n-3}(z)(\tau) \chi(\tau)^{2} l(z)(\tau)(l(z)(\sigma))^{2}\right. \\
& \left.\quad-\chi(\tau)^{n-2} l_{n-3}(z)(\sigma)(l(z)(\tau))^{2} l(z)(\sigma)\right)+\cdots .
\end{aligned}
$$

The assumptions of the theorem imply that

$$
\sum_{i=1}^{N} n_{i} l_{n}\left(z_{i}\right)(\tau \sigma)=\sum_{i=1}^{N} n_{i} l_{n}\left(z_{i}\right)(\tau)+\chi(\tau)^{n} \sum_{i=1}^{N} n_{i} l_{n}\left(z_{i}\right)(\sigma) .
$$

The $l$-adic polylogarithm $l_{n}(z)_{p}$ depends on a choice of a $\mathbf{Q}_{l}$-path from $\overrightarrow{01}$ to $z$. We have the following elementary result.

LEMMA 11.0.10. Let $p$ be a $\mathbf{Q}_{l}$-path from $\overrightarrow{01}$ to $z$ and let $S \in \pi_{1}\left(V_{\bar{K}}, \overrightarrow{01}\right)$ $\otimes \mathbf{Q}$. If $S \equiv x^{\alpha} \cdot y^{\beta} \bmod \Gamma^{2}\left(\pi_{1}\left(V_{\bar{K}} ; \overrightarrow{01}\right) \otimes \mathbf{Q}\right)$ then $l(z)_{p S}=l(z)_{p}+\alpha(\chi-1)$ and $l_{1}(z)_{p S}=l_{1}(z)_{p}+\beta(\chi-1)$.

Proof. We have $\mathfrak{f}_{p S}(\sigma)=S^{-1} \cdot \mathfrak{f}_{p}(\sigma) \cdot \sigma(S)$. Hence $\Lambda_{p S}(\sigma)=k(S)^{-1}$. $\Lambda_{p}(\sigma) \cdot k(\sigma(S))$. Let $S=x^{\alpha} \cdot y^{\beta} \cdot e_{2}^{\beta_{2}} \cdot e_{3}^{\beta_{3}} \cdot f_{3} \cdot e_{4}^{\beta_{4}} \ldots$. Therefore $\log \Lambda_{p S}(\sigma)=$ $\left(-\log \left(e^{\alpha X} \cdot e^{\beta Y} \cdot\left(e^{X} \cdot e^{Y} \cdot e^{-X} \cdot e^{-Y}\right)^{\beta_{2}} \cdots\right)\right) \bigcirc \log \Lambda_{p}(\sigma) \bigcirc \log \left(e^{\alpha \chi(\sigma) X}\right.$. $\left.e^{\beta \chi(\sigma) Y} \cdot\left(e^{\chi(\sigma) X} \cdot e^{\chi(\sigma) Y} \cdot e^{-\chi(\sigma) X} \cdot e^{-\chi(\sigma) Y}\right)^{\beta_{2}} \cdots\right) \equiv-\alpha X-\beta Y+l(z)_{p}(\sigma) X+$ $l_{1}(z)_{p}(\sigma) Y+\alpha \chi(\sigma) X+\beta \chi(\sigma) Y \bmod \Gamma^{2} L(X, Y)$. The lemma follows from the congruence.

THEOREM 11.0.11. Let $z_{i} \in V(K)$, let $p_{i} \in \pi\left(V_{\bar{K}} ; z_{i}, \overrightarrow{01}\right) \otimes \mathbf{Q}$ and let $n_{i} \in \mathbf{Q}$ for $i=1, \ldots, N$. Let $\mathcal{S}$ be a subgroup of $K^{*} \otimes \mathbf{Q}$ generated by $z_{i}$ and $1-z_{i}$ for $i=1, \ldots, N$. Assume that

i) the map $\varphi: \mathcal{S} \rightarrow Z^{1}\left(G_{K} ; \mathbf{Q}_{l}(1)\right)$ given by $\varphi\left(z_{i}\right)=l\left(z_{i}\right)_{p_{i}}$ and $\varphi(1-$ $\left.z_{i}\right)=l_{1}\left(z_{i}\right)_{p_{i}}$ is well defined and it is a homomorphism;

ii) $\sum_{i=1}^{N} n_{i} \nu_{1}\left(z_{i}\right) \cdots \nu_{n-2}\left(z_{i}\right)\left(z_{i}\right) \wedge\left(1-z_{i}\right)=0$ in $(\mathcal{S} \wedge \mathcal{S}) \otimes \mathbf{Q}_{l}$ for any homomorphisms $\nu_{1}, \ldots, \nu_{n-2}$ from $\mathcal{S}$ to $\mathbf{Q}_{l}$; 
iii) $\sum_{i=1}^{N} n_{i} \cdot \nu_{1}\left(z_{i}\right)^{\alpha} \cdot \nu_{2}\left(z_{i}\right)^{\beta} \cdot l_{k}\left(z_{i}\right)(\sigma)=0$ for any homomorphisms $\nu_{1}$ and $\nu_{2}$ from $\mathcal{S}$ to $\mathbf{Q}_{l}$, for any $\sigma \in G_{K}$, for $k=2, \ldots, n-1$ and for any $\alpha$ and $\beta$ such that $\alpha+\beta=n-k$.

Then $\sum_{i=1}^{N} n_{i} l_{n}\left(z_{i}\right)_{p_{i}}$ is a cocycle on $G_{K}$ with values in $\mathbf{Q}_{l}(n)$.

Proof. Let us fix $\tau \in G_{K}$. The map $\mathcal{S} \rightarrow \mathbf{Q}_{l}(1)$ given by $s \rightarrow \varphi(s)(\tau)$ $\left(z_{i} \rightarrow l\left(z_{i}\right)(\tau)\right)$ is a homomorphism. Let us fix $\tau, \sigma \in G_{K}$. The map $\mathcal{S} \otimes \mathcal{S} \rightarrow \mathbf{Q}_{l}(2), x \otimes y \rightarrow \varphi(x)(\tau) \cdot \varphi(y)(\sigma)-\varphi(x)(\sigma) \cdot \varphi(y)(\tau)\left(z_{i} \otimes\left(1-z_{i}\right) \rightarrow\right.$ $\left.l\left(z_{i}\right)(\tau) \cdot l_{1}\left(z_{i}\right)(\sigma)-l\left(z_{i}\right)(\sigma) \cdot l_{1}\left(z_{i}\right)(\tau)\right)$ factors through $\mathcal{S} \wedge \mathcal{S}$. Hence the theorem follows from Theorem 11.0.9.

COROLlaRY 11.0.12. Let $\xi_{m}$ be a m-th root of 1 different from 1 .

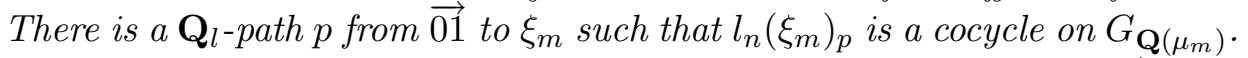
If $l$ does not divide $m$ then one can choose the path $p$ in $\pi\left(V_{\overline{\mathbf{Q}}} ; \xi_{m}, \overrightarrow{01}\right)$.

Proof. Let $m=l^{k_{0}} \cdot r$, where $l$ does not divide $r$. Let $q$ be a path from $\overrightarrow{01}$ to $\xi_{m}$. There are $\alpha, \beta$ and $\gamma$ in $\mathbf{Z}_{l}$ such that $\left(\xi_{l^{k_{0}+n}}^{\alpha} \cdot \xi_{r}^{\beta / l^{n}} \cdot \xi_{l^{n}}^{\gamma}\right)_{n \in \mathbf{N}}$ is a compatible family of $l^{n}$-th roots of $\xi_{m}$ determined by the path $q$. Hence $l\left(\xi_{m}\right)_{q}=\left(\frac{\alpha}{k_{0}}+\gamma\right)(\chi-1)$. Lemma 11.0.10 implies that there is a $\mathbf{Q}_{l}$-path $p$ from $\overrightarrow{01}$ to $\xi_{m}$ such that $l\left(\xi_{m}\right)_{p}=0$. Theorem 11.0.9 implies that $l_{n}\left(\xi_{m}\right)_{p}$ is a cocycle. Observe that if $k_{0}=0$ then one can choose $p$ in $\pi\left(V_{\overline{\mathbf{Q}}} ; \xi_{m}, \overrightarrow{01}\right)$.

The classical polylogarithms are iterated integrals defined by $\int_{0}^{z} \frac{d z}{1-z}, \frac{d z}{z}$, $\ldots, \frac{d z}{z}$. The iterated integral $\int_{a}^{b} \frac{d z}{1-z}, \frac{d z}{z}, \ldots, \frac{d z}{z}$ can be express by classical polylogarithms. Now we shall define a normalized analog of the iterated integral $\int_{a}^{b} \frac{d z}{1-z}, \frac{d z}{z}, \ldots, \frac{d z}{z}$.

Let $z, v \in \hat{V}(K)$. Let $q$ be a path from $\overrightarrow{01}$ to $v$ and let $p$ be a path from $v$ to $z$. We shall define relative polylogarithms $l_{n}(z, v)$. Let us set $x_{1}:=$ $q \cdot x \cdot q^{-1}, y_{1}:=q \cdot y \cdot q^{-1}$. Observe that $x_{1}, y_{1}$ are generators of $\pi_{1}\left(V_{\bar{K}} ; v\right)$. Let $G_{n+1} \subset \pi_{1}\left(V_{\bar{K}} ; \overrightarrow{01}\right)$ (resp. $\left.G_{n+1}^{\prime} \subset \pi_{1}\left(V_{\bar{K}} ; v\right)\right)$ be a closed normal subgroup generated by $\Gamma^{n+1} \pi_{1}\left(V_{\bar{K}} ; \overrightarrow{01}\right)$ (resp. $\left.\Gamma^{n+1} \pi_{1}\left(V_{\bar{K}} ; v\right)\right)$ and all commutators which contain $y$ (resp. $y_{1}$ ) at least twice. Let $\pi:=\pi_{1}\left(V_{\bar{K}} ; \overrightarrow{01}\right) / G_{n+1}$ and $\pi^{\prime}:=\pi_{1}\left(V_{\bar{K}} ; v\right) / G_{n+1}^{\prime}$.

It follows from Proposition 2.2.1 that the action of $G_{K}$ on $\pi^{\prime}$ is given by

$$
\sigma\left(x_{1}\right)=\left(q \cdot \mathfrak{f}_{q}(\sigma) \cdot q^{-1}\right) \cdot x_{1}^{\chi(\sigma)} \cdot\left(q \cdot\left(\mathfrak{f}_{q}(\sigma)\right)^{-1} \cdot q^{-1}\right) \bmod G_{m+1}^{\prime}
$$


and

$$
\sigma\left(y_{1}\right)=\left(q \cdot \mathfrak{f}_{q}(\sigma) \cdot q^{-1}\right) \cdot y_{1}^{\chi(\sigma)} \cdot\left(q \cdot\left(\mathfrak{f}_{q}(\sigma)\right)^{-1} \cdot q^{-1}\right) \bmod G_{m+1}^{\prime} .
$$

LEMmA 11.0.13. The action of $G_{K}$ on $\pi_{1}\left(V_{\bar{K}} ; v\right)$ induced from the action on the torsor $\pi\left(V_{\bar{Q}} ; z, v\right)$ by the isomorphism $t_{p}$ (see Part I Section 1$)$ is given by

$$
\sigma_{p}(w)=\left(q \cdot \mathfrak{f}_{p q}(\sigma) \cdot q^{-1}\right) \cdot \bar{\sigma}(w) \cdot\left(q \cdot\left(\mathfrak{f}_{q}(\sigma)\right)^{-1} \cdot q^{-1}\right) \bmod G_{n+1}^{\prime},
$$

where $\bar{\sigma}\left(x_{1}\right)=x_{1}^{\chi(\sigma)}, \bar{\sigma}\left(y_{1}\right)=y_{1}^{\chi(\sigma)}$ and $\bar{\sigma}$ is continous and multiplicative.

Proof. The formula for $\sigma_{p}(w)$ follows from Lemma 1.0.2 and Lemma 1.0.6.

Let $I$ be the augmentation ideal of $\mathbf{Q}_{l}\{\{X, Y\}\}$ and let $J_{n+1}$ be a closed ideal of $\mathbf{Q}_{l}\{\{X, Y\}\}$ generated by $I^{n+1}$ and all monomials which contain $Y$ at least twice. We define two maps

$$
\begin{aligned}
& k: \pi_{1}\left(V_{\bar{K}} ; \overrightarrow{01}\right) \longrightarrow \mathbf{Q}_{l}\{\{X, Y\}\} / J_{n+1} \quad \text { and } \\
& k^{\prime}: \pi_{1}\left(V_{\bar{K}} ; v\right) \longrightarrow \mathbf{Q}_{l}\{\{X, Y\}\} / J_{n+1}
\end{aligned}
$$

by $k(x)=e^{X}, k(y)=e^{Y}$ and $k^{\prime}\left(x_{1}\right)=e^{X}, k^{\prime}\left(y_{1}\right)=e^{Y}$.

Let ()$_{p}: G_{K} \rightarrow G L\left(\mathbf{Q}_{l}\{\{X, Y\}\} / J_{n+1}\right)$ be the action of $G_{K}$ induced from the action of $G_{K}$ on the torsor $\pi\left(V_{\overline{\mathbf{Q}}} ; z, v\right)$ by the isomorphism $t_{p}$ and the embedding $k^{\prime}$.

Let us set

$$
\psi_{p}(\sigma):=\sigma_{p} \circ \rho\left(\chi(\sigma)^{-1}\right) .
$$

We recall that $E_{1}:=Y$ and $E_{k+1}:=\left[E_{k}, X\right]$ for $k=1, \ldots, n-1$. Then any Lie element of $\mathbf{Q}_{l}\{\{X, Y\}\} / J_{n+1}$ is a linear combination with $\mathbf{Q}_{l}$ coefficients of $X, E_{1}, \ldots, E_{n}$. If $g \in \pi^{\prime}$ then $\log k^{\prime}(g)$ is a Lie element of $\mathbf{Q}_{l}\{\{X, Y\}\} / J_{n+1}$.

Definition 11.0.14. Let $\sigma \in G_{K}$. We set

$$
\left(\log \psi_{p}(\sigma)\right)(1)=l(z, v)_{p}(\sigma) X+\sum_{k=1}^{n} l_{k}(z, v)_{p}(\sigma) E_{k} .
$$

Proposition 11.0.15. We have

$$
l_{n}(z, v)_{p}=l_{n}(z)_{p q}-l_{n}(v)_{q} .
$$


Proof. Observe that $k^{\prime}\left(q \cdot \mathfrak{f}_{p q}(\sigma) \cdot q^{-1}\right)=k\left(\mathfrak{f}_{p q}(\sigma)\right)=\Lambda_{p q}(\sigma)$ and $k^{\prime}(q$. $\left.\left(\mathfrak{f}_{q}(\sigma)\right)^{-1} \cdot q^{-1}\right)=k\left(\left(\mathfrak{f}_{q}(\sigma)\right)^{-1}\right)=\left(\Lambda_{q}(\sigma)\right)^{-1}$. Let $\sigma \in G_{K}$. It follows from Lemma 11.0.13 that

$$
\psi_{p}(\sigma)=L_{\Lambda_{p q}(\sigma)} \circ R_{\left(\Lambda_{q}(\sigma)\right)^{-1}}
$$

This implies that

$$
\log \psi_{p}(\sigma)=L_{\log \Lambda_{p q}(\sigma)} \bigcirc R_{-\log \Lambda_{q}(\sigma)} .
$$

The operators $L_{\log \Lambda_{p q}(\sigma)}$ and $R_{-\log \Lambda_{q}(\sigma)}$ commute. Hence $\log \psi_{p}(\sigma)=$ $L_{\log \Lambda_{p q}(\sigma)}+R_{-\log \Lambda_{q}(\sigma)}$. This implies the proposition.

Corollary 11.0.16. We have

$$
l_{n}(z, \overrightarrow{01})_{p}=l_{n}(z)_{p} .
$$

Proof. It follows from Proposition 11.0.15 that $l_{n}(z, \overrightarrow{01})_{p}=l_{n}(z)_{p}-$ $l_{n}(\overrightarrow{01})_{c}$, where $c$ is a constant path. For such a path $l_{n}(\overrightarrow{01})_{c}=0$.

Remark. The relative polylogarithm $l_{n}(z, v)$ is the function $a_{p}^{E_{n}}$ from Section 5 . Hence the $l$-adic polylogarithm $l_{n}(z)_{p}$ is also a special case of $l$-adic iterated integrals defined in Section 5.

We finish this subsection with a result expressing coefficiets of $\mathfrak{f}_{p}$ in degree one for an arbitrary $X$ by $l$-adic logarithms.

Proposition 11.0.17. Let $X=\mathbf{P}_{K}^{1} \backslash\left\{a_{1}, \ldots, a_{n}, \infty\right\}$, let $z, v \in \hat{X}(K)$ and let $p$ be a path from $v$ to $z$. Let $g_{i}: X \rightarrow \mathbf{P}_{K}^{1} \backslash\{0, \infty\}$ be given by $g_{i}(z)=z-a_{i}$ for $i=1, \ldots, n$. Then

$\mathfrak{f}_{p} \equiv x_{1}^{l\left(z-a_{1}\right)_{g_{1}(p) \cdot q_{1}}-l\left(v-a_{1}\right)_{q_{1}}} \ldots x_{n}^{l\left(z-a_{n}\right)_{g_{n}(p) \cdot q_{n}}-l\left(v-a_{n}\right)_{q_{n}}} \bmod \Gamma^{2} \pi_{1}\left(X_{\bar{K}} ; v\right)$, where $q_{i}$ is any path from $\overrightarrow{01}$ to $v-a_{i}$ on $\mathbf{P}_{\bar{K}}^{1} \backslash\{0, \infty\}$ for $i=1, \ldots, n$.

Proof. Without loss of generality we can suppose that $X=\mathbf{P}_{K}^{1} \backslash\{a, \infty\}$ and $g: X \rightarrow \mathbf{P}_{K}^{1} \backslash\{0, \infty\}$ is given by $g(z)=z-a$. Let $p$ be a path from $v$ to $z$ on $X_{\bar{K}}$. Then $g(p)$ is a path from $v-a$ to $z-a$ on $\mathbf{P}_{K}^{1} \backslash\{0, \infty\}$. Let $q$ be any path from $\overrightarrow{01}$ to $v-a$ on $\mathbf{P}_{\bar{K}}^{1} \backslash\{0, \infty\}$. We have

$$
\mathfrak{f}_{g(p) \cdot q}=q^{-1} \cdot \mathfrak{f}_{g(p)} \cdot q \cdot \mathfrak{f}_{q}=q^{-1} \cdot g_{*}\left(\mathfrak{f}_{p}\right) \cdot q \cdot \mathfrak{f}_{q} .
$$

It follows from Corollary 11.0.7 that

$$
x^{l(z-a)_{g(p) \cdot q}}=q^{-1} \cdot g_{*}\left(\mathfrak{f}_{p}\right) \cdot q \cdot x^{l(v-a)_{q}},
$$

where $x$ is a loop around 0 . Hence we get that $\mathfrak{f}_{p}=x_{a}^{l(z-a)_{g(p) \cdot q}-l(v-a)_{q}}$, where $g_{*}\left(x_{a}\right)=q \cdot x \cdot q^{-1}$. 
11.1. In this subsection we shall study functional equations of $l$-adic polylogarithms. We shall prove the distribution relation and the Abel five term equation for $l$-adic dilogarithms. We shall show that $l$-adic dilogarithms satisfy these functional equations without lower degree terms.

We start with the discussion of the $l$-adic analog of the functional equation

$$
\log (x \cdot y)=\log x+\log y
$$

of the classical logarithm.

Proposition 11.1.0. Let $\zeta, y \in \mathbf{P}^{1}(K) \backslash\{0, \infty\}$. Then there exist paths $\gamma$ from $\overrightarrow{01}$ to $\zeta, \delta$ from $\overrightarrow{01}$ to $y$ and $\varphi$ from $\overrightarrow{01}$ to $y \cdot \zeta$ such that

$$
l(y \cdot \zeta)_{\varphi}=l(y)_{\delta}+l(\zeta)_{\gamma}
$$

on $G_{K}$.

Proof. Let $g: \mathbf{P}_{K}^{1} \backslash\{0, \infty\} \rightarrow \mathbf{P}_{K}^{1} \backslash\{0, \infty\}$ be given by $g(z)=y \cdot z$. Let $p$ be a path from $\overrightarrow{01}$ to $\zeta$. Then $g(p)$ is a path from $\overrightarrow{0 y}$ to $y \cdot \zeta$. We recall that $x$ is a standard generator of $\pi_{1}\left(\mathbf{P}_{\bar{K}}^{1} \backslash\{0, \infty\}, \overrightarrow{01}\right)$. Let us fix a path $q$ from $\overrightarrow{01}$ to $\overrightarrow{0 y}$. Let us set $x^{\prime}=q \cdot x \cdot q^{-1}$. Then $x^{\prime}$ is a generator of $\pi_{1}\left(\mathbf{P}_{\bar{K}}^{1} \backslash\{0, \infty\}, \overrightarrow{0 y}\right)$. Observe that $g_{*}(x)=x^{\prime}$. It follows from Corollary 11.0.7 that

$$
\mathfrak{f}_{p}(\sigma)=x^{l(\zeta)_{p}(\sigma)} \quad \text { and } \quad \mathfrak{f}_{g(p) \cdot q}(\sigma)=x^{l(y \cdot \zeta)_{g(p) \cdot q}(\sigma)} .
$$

On the other side we have

$$
\begin{aligned}
\mathfrak{f}_{g(p) \cdot q}(\sigma) & =q^{-1} \cdot \mathfrak{f}_{g(p)}(\sigma) \cdot q \cdot \mathfrak{f}_{q}(\sigma)=q^{-1} \cdot g_{*}\left(\mathfrak{f}_{p}(\sigma)\right) \cdot q \cdot \mathfrak{f}_{q}(\sigma) \\
& =x^{l(\zeta)_{p}(\sigma)} \cdot x^{l(\overrightarrow{0 y})_{q}(\sigma)}=x^{l(\zeta)_{p}(\sigma)+l(\overrightarrow{0 y})_{q}(\sigma)} .
\end{aligned}
$$

Comparing exponents we get

$$
l(y \cdot \zeta)_{g(p) \cdot q}=l(\zeta)_{p}+l(\overrightarrow{0 y})_{q}
$$

Let $t$ be the canonical path from $\overrightarrow{0 y}$ to $y$. Then $t \cdot q$ is a path from $\overrightarrow{01}$ to $y$ (see Picture 2).

We have $x^{l(y)_{t \cdot q}(\sigma)}=\mathfrak{f}_{t \cdot q}(\sigma)=q^{-1} \cdot \mathfrak{f}_{t}(\sigma) \cdot q \cdot \mathfrak{f}_{q}(\sigma)=q^{-1} \cdot \mathfrak{f}_{t}(\sigma) \cdot q \cdot x^{l(\overrightarrow{0 y})_{q}(\sigma)}$. It rests to calculate $\mathfrak{f}_{t}(\sigma)$. Without loss of generality we can suppose that $y=1$ and $t$ is the canonical path from $\overrightarrow{01}$ to 1 . Then it is clear that $\mathfrak{f}_{t}(\sigma)=1$. Hence $l(\overrightarrow{0 y})_{q}=l(y)_{t \cdot q}$. This finishes the proof of the proposition. 


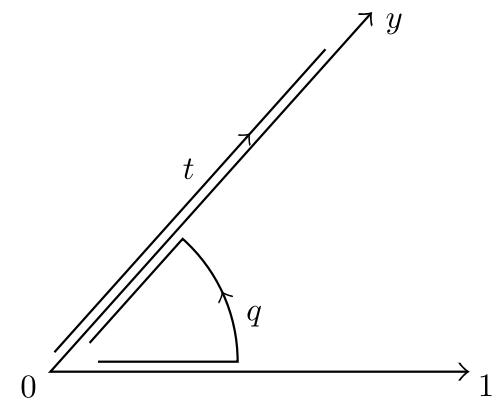

Picture 2

Now we shall discuss the $l$-adic analog of the functional equation

$$
L i_{2}\left(z^{m}\right)=m\left(\sum_{i=0}^{m} L i_{2}\left(\xi_{m}^{i} z\right)\right)
$$

of the classical dilogarithm. Let $Y=\mathbf{P}_{\mathbf{Q}\left(\mu_{m}\right)}^{1} \backslash\left\{0, \mu_{m}, \infty\right\}$ and $V=\mathbf{P}_{\mathbf{Q}\left(\mu_{m}\right)}^{1} \backslash$ $\{0,1, \infty\}$. We choose generators $x^{\prime}, y_{0}, \ldots, y_{m-1}$ of $\pi_{1}\left(Y_{\overline{\mathbf{Q}}}, \overrightarrow{01}\right)$ as on the picture.

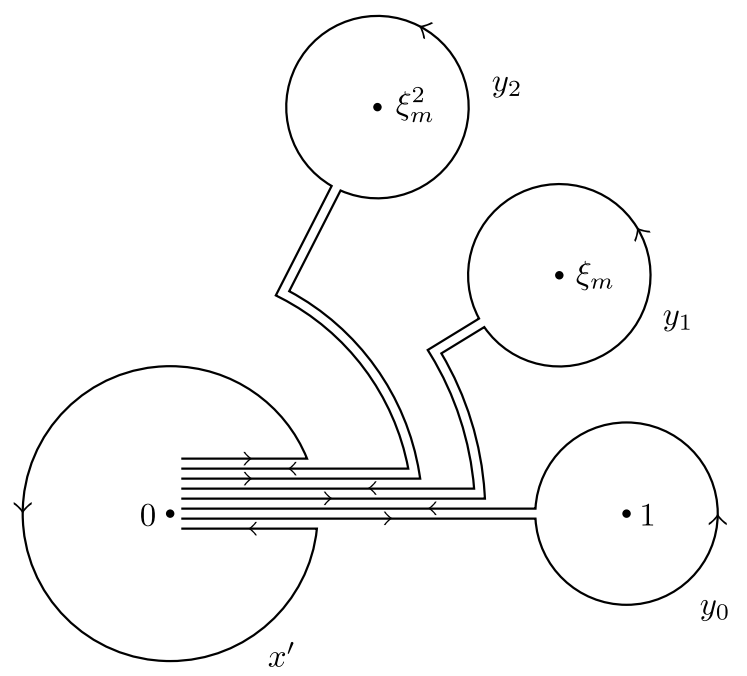

Picture 3

Let $f: Y \rightarrow V$ be given by $f(z)=z^{m}$. We have $f_{*}\left(x^{\prime}\right)=x^{m}, f_{*}\left(y_{0}\right)=y$ and $f_{*}\left(y_{i}\right)=x^{-i} \cdot y \cdot x^{i}$. Let $z \in \hat{Y}\left(\mathbf{Q}\left(\mu_{m}\right)\right)$ and let $p$ be a path from $\overrightarrow{01}$ 
to $z$. We define functions $\lambda(z), \mu_{0}(z), \ldots, \mu_{m-1}(z), \nu_{0}(z), \ldots, \nu_{m-1}(z)$ from $G_{\mathbf{Q}\left(\mu_{m}\right)}$ to $\mathbf{Z}_{l}$ by the following congruence

$$
\begin{aligned}
\mathfrak{f}_{p} \equiv x^{\prime \lambda(z)} \cdot y_{0}^{\mu_{0}(z)} \cdot y_{1}^{\mu_{1}(z)} \cdots \cdots y_{m-1}^{\mu_{m-1}(z)} \\
\cdot\left(y_{0}, x^{\prime}\right)^{\nu_{0}(z)} \cdots \cdots\left(y_{m-1}, x^{\prime}\right)^{\nu_{m-1}(z)} \\
\cdot \prod_{i<j}\left(y_{i}, y_{j}\right)^{\alpha_{i j}(z)} \bmod \Gamma^{3} \pi_{1}\left(Y_{\overline{\mathbf{Q}}} ; \overrightarrow{01}\right) .
\end{aligned}
$$

Observe that $f(p)$ is a path from $\overrightarrow{01}$ to $z^{m}$. Hence we have

$$
\mathfrak{f}_{f(p)} \equiv x^{\kappa_{z m}^{0}} \cdot y^{\kappa_{z}^{1}} \cdot(y, x)^{\kappa_{z m}^{2}} \bmod \Gamma^{3} \pi_{1}\left(V_{\overline{\mathbf{Q}}} ; \overrightarrow{01}\right)
$$

(see Definition 11.0.3).

LEMMA 11.1.2. We have $\kappa_{z^{m}}^{0}=m \lambda(z), \kappa_{z^{m}}^{1}=\mu_{0}(z)+\mu_{1}(z)+\cdots+$ $\mu_{m-1}(z)$ and $\kappa_{z^{m}}^{2}=m\left(\nu_{0}(z)+\cdots+\nu_{m-1}(z)\right)+\mu_{1}(z)+\cdots+i \mu_{i}(z)+\cdots+$ $(m-1) \mu_{m-1}(z)$.

Proof. We have

$$
\begin{aligned}
f_{*} \mathfrak{f}_{p} \equiv & x^{m \lambda(z)} \cdot y^{\mu_{0}(z)} \cdot x^{-1} \cdot y^{\mu_{1}(z)} \cdot x \cdot \cdots \\
\cdot x^{-(m-1)} \cdot y^{\mu_{m-1}(z)} \cdot x^{m-1} \cdot(y, x)^{m\left(\nu_{0}(z)+\cdots+\nu_{m-1}(z)\right)} & \quad \bmod \Gamma^{3} \pi_{1}\left(V_{\overline{\mathbf{Q}}} ; \overrightarrow{01}\right) .
\end{aligned}
$$

Observe that $f_{*} \mathfrak{f}_{p}=\mathfrak{f}_{f(p)}$. Comparing exponents of $f_{*} \mathfrak{f}_{p}$ and $\mathfrak{f}_{f(p)}$ we get the equalities of the lemma.

Let $q_{i}$ be a path from $\overrightarrow{0 \xi_{m}^{i}}$ to $\overrightarrow{01}$ as on Picture 4 .

Let us set $x_{i}:=q_{i}^{-1} \cdot x^{\prime} \cdot q_{i}$ and $y_{k}^{(i)}:=q_{i}^{-1} \cdot y_{k} \cdot q_{i}$. Let $f_{i}: Y \rightarrow V$ be given by $f_{i}(z)=\xi_{m}^{-i} \cdot z$. Observe that $\left(f_{i}\right)_{*} \overrightarrow{0 \xi_{m}^{i}}=\overrightarrow{01},\left(f_{i}\right)_{*}\left(x_{i}\right)=x$, $\left(f_{i}\right)_{*}\left(y_{i}^{(i)}\right)=y$ and $\left(f_{i}\right)_{*}\left(y_{k}^{(i)}\right)=1$ for $k \neq i$.

LEMMA 11.1.3. We have

$$
\kappa_{\xi_{m}^{-i} z}^{0}=\lambda(z)+\frac{i}{m}(1-\chi), \quad \kappa_{\xi_{m}^{-i} z}^{1}=\mu_{i}(z) \text { and } \kappa_{\xi_{m}^{-i} z}^{2}=\nu_{i}(z)+\frac{i}{m}(1-\chi) \mu_{i}(z) .
$$




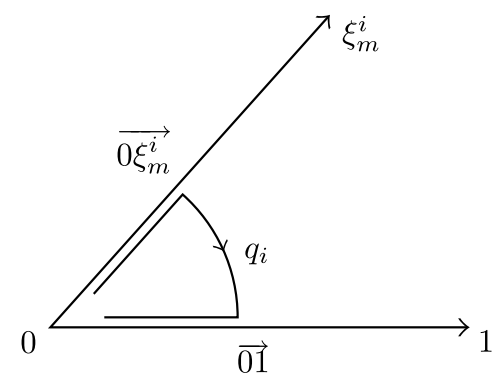

Picture 4

Proof. $f_{i}\left(p q_{i}\right)$ is a path from $\overrightarrow{01}$ to $\xi_{m}^{-i} z$. Hence we have

$$
\mathfrak{f}_{f_{i}\left(p q_{i}\right)} \equiv x^{\kappa^{0}} \xi_{m}^{-i} z \cdot y^{\kappa^{1}} \xi_{m}^{-i} z \cdot(y, x)^{\kappa^{2}} \xi_{m}^{-i} z \bmod \Gamma^{3} \pi_{1}\left(V_{\overline{\mathbf{Q}}} ; \overrightarrow{01}\right),
$$

by the Definition 11.0.3. On the other side

$$
\left(f_{i}\right)_{*} \mathfrak{f}_{p q_{i}}=\left(f_{i}\right)_{*}\left(q_{i}^{-1} \cdot \mathfrak{f}_{p} \cdot q_{i}\right) \cdot\left(f_{i}\right)_{*}\left(\mathfrak{f}_{q_{i}}\right) .
$$

Hence it follows from (11.1.1) that

$$
\begin{aligned}
\left(f_{i}\right)_{*} \mathfrak{f}_{p q_{i}} & \equiv x^{\lambda(z)} \cdot y^{\mu_{i}(z)} \cdot(y, x)^{\nu_{i}(z)} \cdot x^{\frac{i}{m}(1-\chi)} \\
& \equiv x^{\lambda(z)+\frac{i}{m}(1-\chi)} \cdot y^{\mu_{i}(z)} \cdot(y, x)^{\nu_{i}(z)+\frac{i}{m}(1-\chi) \mu_{i}(z)} \bmod \Gamma^{3} \pi_{1}\left(V_{\overline{\mathbf{Q}}} ; \overrightarrow{01}\right)
\end{aligned}
$$

We have the identity

$$
\left(f_{i}\right)_{*} \mathfrak{f}_{p q_{i}}=\mathfrak{f}_{f_{i}\left(p q_{i}\right)} .
$$

Hence comparing exponents of $\left(f_{i}\right)_{*} \mathfrak{f}_{p q_{i}}$ and $\mathfrak{f}_{f_{i}\left(p q_{i}\right)}$ we get the equalities of the lemma.

Proposition 11.1.4. Let $l_{2}\left(z^{m}\right)$ be calculated along the path $f(p)$ and let $l_{2}\left(\xi_{m}^{-i} z\right)$ be calculated along the $\mathbf{Q}_{l}$-path $f_{i}\left(p q_{i}\right) \cdot x^{\frac{i}{m}}$ for $i=0,1, \ldots, m-1$. Then we have

$$
l_{2}\left(z^{m}\right)=m\left(\sum_{i=0}^{m-1} l_{2}\left(\xi_{m}^{-i} z\right)\right) .
$$

Proof. It follows from Corollary 11.0.7 that

$$
l_{2}(z)_{p}(\sigma)=\kappa_{z}^{2}(\sigma)_{p}-\frac{1}{2} \kappa_{z}^{0}(\sigma)_{p} \cdot \kappa_{z}^{1}(\sigma)_{p}
$$


Hence it follows from Lemma 11.1.2 that

$$
l_{2}\left(z^{m}\right)_{f(p)}=m\left(\sum_{i=0}^{m-1} \nu_{i}(z)\right)+\sum_{i=0}^{m-1} i \mu_{i}(z)-\frac{1}{2} m \lambda(z)\left(\sum_{i=0}^{m-1} \mu_{i}(z)\right) .
$$

Let us calculate $l_{2}\left(\xi_{m}^{-i} z\right) f_{i}\left(p q_{i}\right) \cdot x^{\frac{i}{m}}$. We have

$$
\mathfrak{f}_{f_{i}\left(p q_{i}\right) \cdot x^{\frac{i}{m}}}(\sigma)=x^{-\frac{i}{m}} \cdot \mathfrak{f}_{f_{i}\left(p q_{i}\right)}(\sigma) \cdot x^{\frac{i}{m} \chi(\sigma)} .
$$

Hence it follows from Lemma 11.1.3 that

$$
l_{2}\left(\xi_{m}^{-i} z\right)_{f_{i}\left(p q_{i}\right) x^{\frac{i}{m}}}=\nu_{i}(z)+\frac{i}{m} \mu_{i}(z)-\frac{1}{2} \lambda(z) \mu_{i}(z) .
$$

Comparing formulas for $l_{2}\left(z^{m}\right)_{f(p)}$ and $l_{2}\left(\xi_{m}^{-i} z\right){ }_{f_{i}\left(p q_{i}\right) \cdot x^{\frac{i}{m}}}$ we get

$$
l_{2}\left(z^{m}\right)_{f(p)}=m\left(\sum_{i=0}^{m-1} l_{2}\left(\xi_{m}^{-i} z\right) f_{i}\left(p q_{i}\right) \cdot x^{\frac{i}{m}}\right) .
$$

The classical dilogarithm satisfy the functional equation

$$
\begin{aligned}
& L i_{2}\left(\frac{(1-y) z}{z-1}\right)-L i_{2}(y z)+L i_{2}\left(\frac{(z-1) y}{1-y}\right)-L i_{2}\left(\frac{y}{y-1}\right)+L i_{2}(z) \\
& \quad=\text { lower degree terms. }
\end{aligned}
$$

We shall prove its $l$-adic analog.

Let $V=\mathbf{P}_{K}^{1} \backslash\{0,1, \infty\}$ and let $Y=\mathbf{P}_{K}^{1} \backslash\left\{0,1, \frac{1}{y}, \infty\right\}$, where $y \in$ $K \backslash\{0,1\}$. Let $\xi, \zeta \in \hat{V}(K)$ and let $\pi$ be a path from $\xi$ to $\zeta$ and let $\rho$ be a path from $\overrightarrow{01}$ to $\xi$ (see Picture 5 ).

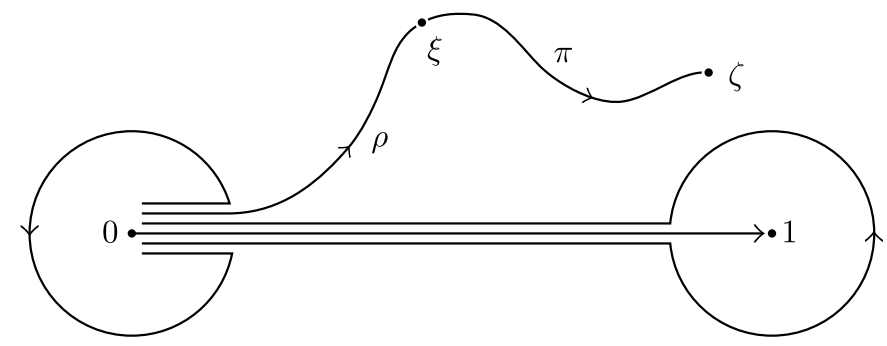

Picture 5 
Let us set

$$
x^{\prime}=\rho \cdot x \cdot \rho^{-1} \quad \text { and } \quad y^{\prime}=\rho \cdot y \cdot \rho^{-1}
$$

where $x, y$ are generators of $\pi_{1}\left(V_{\bar{K}} ; \overrightarrow{01}\right)$ as in 11.0. We define functions $\mathfrak{k}(\pi)$, $\mathfrak{k}_{1}(\pi)$ and $\mathfrak{k}_{2}(\pi)$ from $G_{K}$ to $\mathbf{Z}_{l}$ by the following congruence

$$
\mathfrak{f}_{\pi} \equiv x^{\prime \mathfrak{k}(\pi)} \cdot y^{\prime \mathfrak{k}_{1}(\pi)} \cdot\left(y^{\prime}, x^{\prime}\right)^{\mathfrak{k}_{2}(\pi)} \bmod \Gamma^{3} \pi_{1}\left(V_{\bar{K}} ; \xi\right) .
$$

LEMMA 11.1.5. i) We have

$$
l_{2}(\zeta)_{\pi \rho}-l_{2}(\xi)_{\rho}=\mathfrak{k}_{2}(\pi)-\frac{1}{2} \mathfrak{k}(\pi) \mathfrak{k}_{1}(\pi)-\frac{1}{2} \kappa_{\zeta}^{0} \kappa_{\xi}^{1}+\frac{1}{2} \kappa_{\xi}^{0} \kappa_{\zeta}^{1}
$$

ii) If we replace $\rho$ by $\rho_{1}=\rho \cdot x^{a}$ then in terms of new generators $x^{\prime \prime}=$ $\rho_{1} \cdot x \cdot \rho_{1}^{-1}, y^{\prime \prime}=\rho_{1} \cdot y \cdot \rho_{1}^{-1}$ the triple $\mathfrak{k}(\pi), \mathfrak{k}_{1}(\pi), \mathfrak{k}_{2}(\pi)$ is replaced by the triple $\mathfrak{k}(\pi), \mathfrak{k}_{1}(\pi), \mathfrak{k}_{2}(\pi)+a \mathfrak{k}_{1}(\pi)$.

Proof. It follows from the formula $\mathfrak{f}_{\pi \rho}=\rho^{-1} \mathfrak{f}_{\pi} \rho \cdot \mathfrak{f}_{\rho}$ (see Lemma 1.0.6) that $\Lambda_{\pi \rho}(\sigma)=\Lambda_{\pi}(\sigma) \cdot \Lambda_{\rho}(\sigma)$, where $\Lambda_{\pi}(\sigma)$ is the image of $\mathfrak{f}_{\pi}$ by the embedding of $\pi_{1}\left(V_{\bar{K}} ; \xi\right)$ into $\mathbf{Q}_{l}\{\{X, Y\}\}$ sending $x^{\prime}$ to $e^{X}$ and $y^{\prime}$ to $e^{Y}$. Applying logarithm we get

$$
\log \Lambda_{\pi \rho}(\sigma) \bigcirc\left(-\log \Lambda_{\rho}(\sigma)\right)=\log \Lambda_{\pi}(\sigma)
$$

Comparing coefficient at $[Y, X]$ we get

$$
l_{2}(\zeta)_{\pi \rho}-l_{2}(\xi)_{\rho}=\mathfrak{k}_{2}(\pi)-\frac{1}{2} \mathfrak{k}(\pi) \mathfrak{k}_{1}(\pi)-\frac{1}{2} \kappa_{\zeta}^{0} \kappa_{\xi}^{1}+\frac{1}{2} \kappa_{\xi}^{0} \kappa_{\zeta}^{1}
$$

The second part of the lemma follows from the congruence $y^{\prime \prime}=x^{\prime a} \cdot y^{\prime}$. $x^{\prime-a} \equiv y^{\prime} \cdot\left(y^{\prime}, x^{\prime}\right)^{-a} \bmod \Gamma^{3} \pi_{1}\left(V_{\bar{K}} ; \xi\right)$.

Definition 11.1.6. Let us set

$$
K(\zeta, \xi):=-\kappa_{\zeta}^{0} \kappa_{\xi}^{1}+\kappa_{\xi}^{0} \kappa_{\zeta}^{1}
$$

Observe that $K(\zeta, \xi)$ is a function from $G_{K}$ to $\mathbf{Q}_{l}$. After the restriction to $G_{K\left(\mu_{l} \infty\right)}$ the function $K(\zeta, \xi)$ does not depend on a choice of paths from $\overrightarrow{01}$ to $\xi$ and $\zeta$.

Now we start to look for $l$-adic analog of the 5 -term functional equation of the classical dilogarithm. Let $f(z)=\frac{(1-y) z}{z-1}, g(z)=y z, h(z)=\frac{(z-1) y}{1-y}$ and $k(z)=z$. Observe that $f, g, h$ and $k$ define regular maps from $Y$ to $V$. 


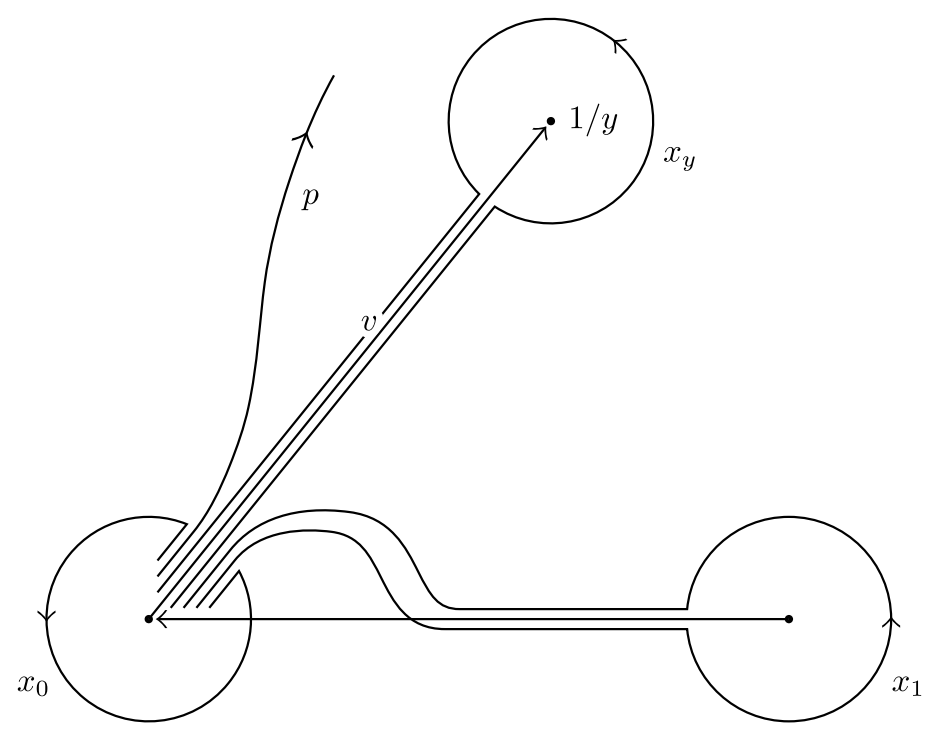

Picture 6

Let $v$ be a tangential base point at 0 corresponding to the local parameter $y z$ at 0 . Let $x_{0}, x_{1}, x_{y}, x_{\infty}$ be geometric generators of $\pi_{1}\left(Y_{\overline{\mathbf{Q}}} ; v\right)$ - loops around $0,1, \frac{1}{y}$ and $\infty$ respectively (see Picture 6 ).

We assume that

$$
x_{\infty} \cdot x_{y} \cdot x_{1} \cdot x_{0}=1
$$

Let $z \in \hat{Y}(K)$ and let $p \in \pi\left(Y_{\bar{K}} ; z, v\right)$. We introduce functions $\lambda(z), \mu(z)$, $\nu(z), \alpha(z), \beta(z)$ and $\gamma(z)$ from $G_{K}$ to $\mathbf{Z}_{l}$ by the following congruence

$$
\begin{array}{r}
\mathfrak{f}_{p} \equiv x_{0}^{\lambda(z)} \cdot x_{1}^{\mu(z)} \cdot x_{y}^{\nu(z)} \cdot\left(x_{1}, x_{0}\right)^{\alpha(z)} \cdot\left(x_{y}, x_{0}\right)^{\beta(z)} \cdot\left(x_{y}, x_{1}\right)^{\gamma(z)} \\
\bmod \Gamma^{3} \pi_{1}\left(Y_{\bar{K}} ; v\right) .
\end{array}
$$

We recall that $f: Y \rightarrow V$ is given by $f(z)=\frac{(1-y) z}{z-1}$. Observe that $f_{*}(v)=w$, where $w$ is a tangential base point at 0 corresponding to the local parameter $\frac{y}{y-1} \cdot z$ at 0 . Let us set $x^{\prime}:=f_{*}\left(x_{0}\right)$ and $y^{\prime}:=f_{*}\left(x_{y}\right)$. Observe that $f(\infty)=1-y$. This implies that $f_{*}\left(x_{\infty}\right)=1$. Therefore $f_{*}\left(x_{1}\right)=y^{\prime-1} \cdot x^{\prime-1}$. Let $q$ be a path from $\overrightarrow{01}$ to $f_{*}(v)$ such that $q \cdot x \cdot q^{-1}=x^{\prime}$ and $q \cdot y \cdot q^{-1}=y^{\prime}$ (see Picture 7).

By the definition of functions $\mathfrak{k}$ and $\mathfrak{k}_{i}$ we have

$$
\mathfrak{f}_{f(p)} \equiv x^{\prime \mathfrak{k}(f(p))} \cdot y^{\prime \mathfrak{k}_{1}(f(p))} \cdot\left(y^{\prime}, x^{\prime}\right)^{\mathfrak{k}_{2}(f(p))} \bmod \Gamma^{3} \pi_{1}\left(V_{\bar{K}}, f_{*}(v)\right) .
$$




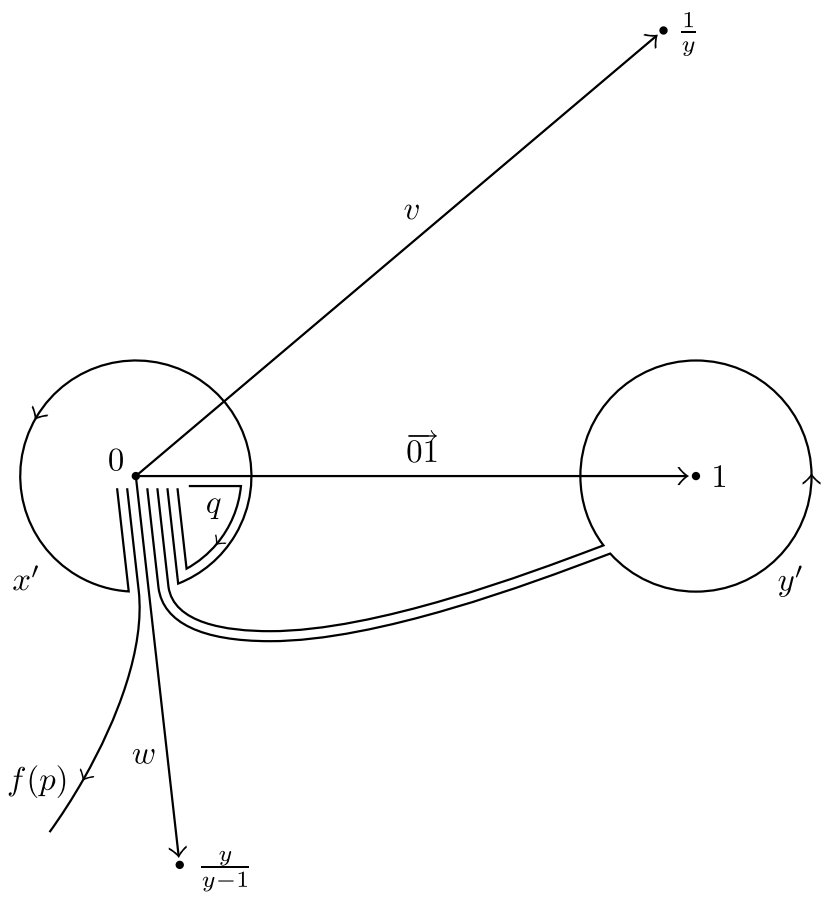

Picture 7

Applying $f_{*}$ to $(11.1 .7)$ we get

$$
\begin{array}{r}
f_{*} \mathfrak{f}_{p} \equiv x^{\prime \lambda(z)-\mu(z)} \cdot y^{\prime \nu(z)-\mu(z)} \cdot\left(y^{\prime}, x^{\prime}\right)^{-\alpha(z)+\beta(z)-\gamma(z)+\frac{1}{2} \mu(z)^{2}+\frac{1}{2} \mu(z)} \\
\bmod \Gamma^{3} \pi_{1}\left(V_{\bar{K}}, f_{*}(v)\right) .
\end{array}
$$

The equality $f_{*} \mathfrak{f}_{p}=\mathfrak{f}_{f(p)}$ implies

$$
\mathfrak{k}(f(p))=\lambda(z)-\mu(z), \quad \mathfrak{k}_{1}(f(p))=\nu(z)-\mu(z)
$$

and

$$
\mathfrak{k}_{2}(f(p))=-\alpha(z)+\beta(z)-\gamma(z)+\frac{1}{2} \mu(z)^{2}+\frac{1}{2} \mu(z) .
$$

We recall that $g: Y \rightarrow V$ is given by $g(z)=y z$. Observe that $g_{*}(v)=$ $\overrightarrow{01}, g_{*}\left(x_{0}\right)=x, g_{*}\left(x_{1}\right)=1$ and $g_{*}\left(x_{y}\right)=y$. Comparing coefficients of $\mathfrak{f}_{g(p)}$ and $g_{*} \mathfrak{f}_{p}$ we get

$$
\mathfrak{k}(g(p))=\lambda(z), \quad \mathfrak{k}_{1}(g(p))=\nu(z), \quad \mathfrak{k}_{2}(g(p))=\beta(z) .
$$


We recall that $h: Y \rightarrow V$ is given by $h(z)=\frac{(z-1) y}{1-y}$. Observe that $h_{*}(v)=\frac{y}{y-1}$. Let us set $x_{h}:=h_{*}\left(x_{1}\right)$ and $y_{h}:=h_{*}\left(x_{y}\right)$. Notice that $h_{*}\left(x_{0}\right)=1$. Let $r$ be a path from $\overrightarrow{01}$ to $\frac{y}{y-1}$ such that $r \cdot x \cdot r^{-1}=x_{h}$ and $r \cdot y \cdot r^{-1}=y_{h}$ (see Picture 8 ).

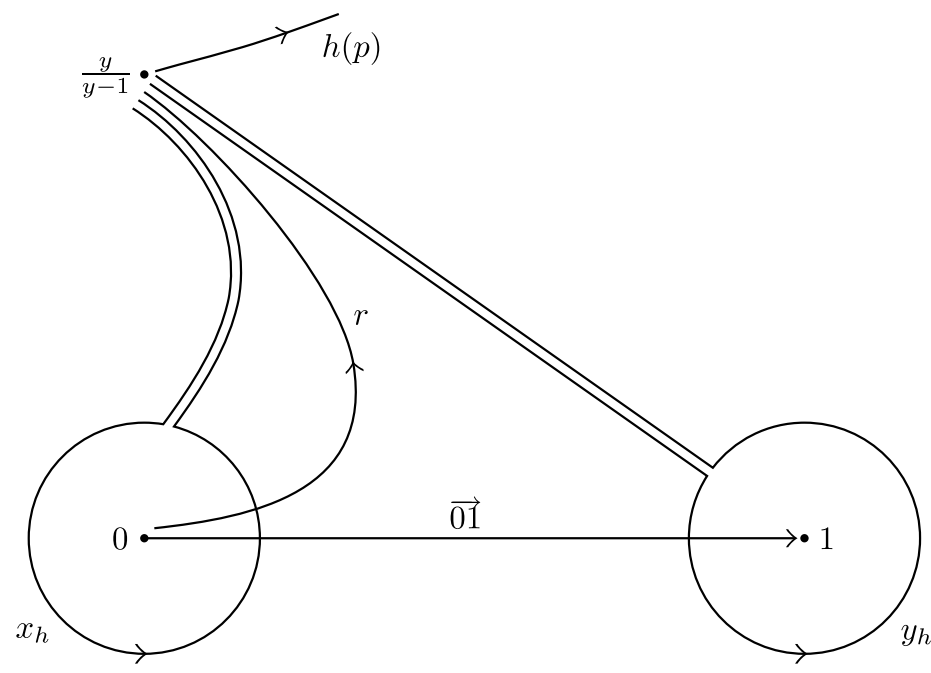

Picture 8

Comparing coefficients of $\mathfrak{f}_{h(p)}$ and $h_{*} \mathfrak{f}_{p}$ we get

$$
\mathfrak{k}(h(p))=\mu(z), \quad \mathfrak{k}_{1}(h(p))=\nu(z), \quad \mathfrak{k}_{2}(h(p))=\gamma(z) .
$$

We recall that $k: Y \rightarrow V$ is given by $k(z)=z$. Observe that $k_{*}(v)=v$. Let us set $x_{k}:=k_{*}\left(x_{0}\right)$ and $y_{k}:=k_{*}\left(x_{1}\right)$. We have $k_{*}\left(x_{y}\right)=1$. Let $s$ be a path from $\overrightarrow{01}$ to $v$ such that

$$
s \cdot x \cdot s^{-1}=x_{k} \quad \text { and } \quad s \cdot y \cdot s^{-1}=y_{k}
$$

(see Picture 9).

Comparing coefficients of $\mathfrak{f}_{k(p)}$ and $k_{*} \mathfrak{f}_{p}$ we get

$$
\mathfrak{k}(k(p))=\lambda(z), \quad \mathfrak{k}_{1}(k(p))=\mu(z), \quad \mathfrak{k}_{2}(k(p))=\alpha(z) .
$$




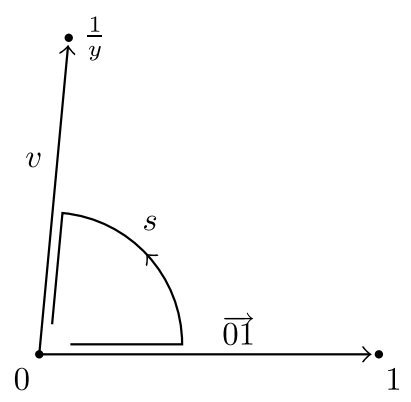

Picture 9

It follows from the equalities (11.1.8)-(11.1.11) that

$$
\begin{aligned}
& \mathfrak{k}_{2}(f(p))-\frac{1}{2} \mathfrak{k}(f(p)) \mathfrak{k}_{1}(f(p))-\mathfrak{k}_{2}(g(p))+\frac{1}{2} \mathfrak{k}(g(p)) \mathfrak{k}_{1}(g(p)) \\
& \quad+\mathfrak{k}_{2}(h(p))-\frac{1}{2} \mathfrak{k}(h(p)) \mathfrak{k}_{1}(h(p))+\mathfrak{k}_{2}(k(p))-\frac{1}{2} \mathfrak{k}(k(p)) \mathfrak{k}_{1}(k(p))=\frac{1}{2} \mu(z) .
\end{aligned}
$$

LEMMA 11.1.13. On $G_{K\left(\mu_{l} \infty\right)}$ we have the following equality

$$
K\left(f(z), f_{*}(v)\right)-K\left(g(z), g_{*}(v)\right)+K\left(h(z), h_{*}(v)\right)+K\left(k(z), k_{*}(v)\right)=0
$$

Proof. We recall that $\kappa_{0}(z)=\kappa_{z}^{0}$ and $\kappa_{1}(z)=\kappa_{z}^{1}$. Hence we have

$$
\begin{gathered}
K\left(f(z), f_{*}(v)\right)-K\left(g(z), g_{*}(v)\right)+K\left(h(z), h_{*}(v)\right)+K\left(k(z), k_{*}(v)\right) \\
=K(f(z), w)-K(g(z), \overrightarrow{01})+K\left(h(z), \frac{y}{y-1}\right)+K(k(z), v) \\
=-\kappa_{0}\left(\frac{(1-y) z}{z-1}\right) \kappa_{1}(w)+\kappa_{0}(w) \kappa_{1}\left(\frac{(1-y) z}{z-1}\right)+\kappa_{0}(y z) \kappa_{1}(\overrightarrow{01}) \\
\quad-\kappa_{0}(\overrightarrow{01}) \kappa_{1}(y z)-\kappa_{0}\left(\frac{(z-1) y}{1-y}\right) \kappa_{1}\left(\frac{y}{y-1}\right) \\
+\kappa_{0}\left(\frac{y}{y-1}\right) \kappa_{1}\left(\frac{(z-1) y}{1-y}\right)-\kappa_{0}(z) \kappa_{1}(v)+\kappa_{0}(v) \kappa_{1}(z) .
\end{gathered}
$$

One checks that $\kappa_{0}(\overrightarrow{0 a})=\kappa_{0}(a)$ and $\kappa_{1}(\overrightarrow{0 a})=0$. The lemma follows from the fact that $\kappa_{0}(x \cdot y)=\kappa_{0}(x)+\kappa_{0}(y)$ and $\kappa_{1}(z)=\kappa_{0}(1-z)$ on $G_{K\left(\mu_{l} \infty\right)}$. 
THEOREM 11.1.14. There are paths $\left(\mathbf{Q}_{2}\right.$-paths if $\left.l=2\right)$ from $\overrightarrow{01}$ to points $\frac{(1-y) z}{z-1}, y z, \frac{(z-1) y}{1-y}, \frac{y}{y-1}$ and $z$ such that on $G_{K\left(\mu_{l} \infty\right)}$ for l-adic dilogarithms calculated along these paths we have

$$
l_{2}\left(\frac{(1-y) z}{z-1}\right)-l_{2}(y z)+l_{2}\left(\frac{(z-1) y}{1-y}\right)-l_{2}\left(\frac{y}{y-1}\right)+l_{2}(z)=0 .
$$

Proof. It follows from Lemma 11.1.5, the equality (11.1.12) and Lemma 11.1.13 that

$$
\begin{aligned}
& l_{2}(f(z))-l_{2}\left(f_{*}(v)\right)-l_{2}(g(z))+l_{2}\left(g_{*}(v)\right) \\
& \quad+l_{2}(h(z))-l_{2}\left(h_{*}(v)\right)+l_{2}(k(z))-l_{2}\left(k_{*}(v)\right)=\frac{1}{2} \mu(z) .
\end{aligned}
$$

To eliminate $\frac{1}{2} \mu(z)$ we replace the path $s$ by $s^{\prime}=s \cdot x^{-1 / 2}$. Then $x_{k}^{\prime}=$ $s^{\prime} \cdot x \cdot s^{\prime-1}=x_{k}$ and $y_{k}^{\prime}=\left(s \cdot x^{-1 / 2}\right) \cdot y \cdot\left(s \cdot x^{-1 / 2}\right)^{-1}=s \cdot y \cdot(y, x)^{1 / 2} \cdot s^{-1}=$ $y_{k} \cdot\left(y_{k}, x_{k}\right)^{1 / 2}$. In terms of generators $x_{k}^{\prime}$ and $y_{k}^{\prime}$ of $\pi_{1}\left(V_{\bar{K}} ; v\right)$ we have

$$
\mathfrak{k}_{2}(k(p))=\alpha(z)-\frac{1}{2} \mu(z) .
$$

Observe that $l_{2}(\overrightarrow{0 a})=0$. Hence we get

$$
l_{2}\left(\frac{(1-y) z}{z-1}\right)-l_{2}(y z)+l_{2}\left(\frac{(z-1) y}{1-y}\right)-l_{2}\left(\frac{y}{y-1}\right)+l_{2}(z)=0
$$

for $l$-adic dilogarithms calculated along the paths $f(p) \cdot q, g(p), h(r) \cdot r, r$ and $k(p) \cdot s \cdot x^{-1 / 2}$ respectively.

It would be interesting to choose paths in such a way that we get the Abel equation on $G_{K}$ without lower degree terms.

11.2. Now we shall discuss functional equations of arbitrary $l$-adic polylogarithms. The next result is a corollary of Theorem 10.0.7. We recall that a subgroup $G_{n+1}$ of $\pi_{1}\left(V_{\overline{\mathbf{Q}}} ; \overrightarrow{01}\right)$ was defined at the end of Subsection 11.0.

We are not able to show that after a suitable choice of paths $l$-adic polylogarithms satisfy functional equations without lower degree terms. We have only the following result.

TheOREM 11.2.1. Let $K$ be a number field and let $V=\mathbf{P}_{K}^{1} \backslash\{0,1, \infty\}$. Let $a_{1}, \ldots, a_{m+1}$ be $K$-points of $\mathbf{P}_{K}^{1}$ and let $Y=\mathbf{P}_{K}^{1} \backslash\left\{a_{1}, \ldots, a_{m+1}\right\}$. Let 
$n_{i} \in \mathbf{Z}$ for $i=1, \ldots, N$ and let $f_{i}: Y \rightarrow V$ be regular maps defined over $K$ for $i=1, \ldots, N$. Let $z, v \in \hat{Y}(K)$. Let us assume that $\sum_{i=1}^{N} n_{i}\left(f_{i}\right)_{*}=0$ in

$$
\operatorname{Hom}\left(\Gamma^{n} \pi_{1}\left(Y_{\bar{K}} ; v\right) / \Gamma^{n+1} \pi_{1}\left(Y_{\bar{K}} ; v\right) ; \Gamma^{n} \pi_{1}\left(V_{\overline{\mathbf{Q}}} ; \overrightarrow{01}\right) / G_{n+1}\right) .
$$

Then we have a functional equation

$$
\sum_{i=1}^{N} n_{i}\left(\mathcal{L}_{n}\left(f_{i}(z)\right)-\mathcal{L}_{n}\left(f_{i}(v)\right)\right)=0
$$

on the subgroup $H_{n}(Y ; z, v)$ of $G_{K}$.

Proof. The theorem follows from Theorem 10.0.7 and Proposition 11.0.15.

Corollary 11.2.2. Let $\xi_{m}$ be a primitive $m$-th root of 1 . Then we have

$$
m^{n-1}\left(\sum_{k=0}^{m-1} \mathcal{L}_{n}\left(\xi_{m}^{k} z\right)\right)=\mathcal{L}_{n}\left(z^{m}\right)
$$

on the subgroup $H_{n}\left(\mathbf{P}_{\mathbf{Q}\left(\mu_{m}\right)}^{1} \backslash\left\{0, \mu_{m}, \infty\right\} ; z, \overrightarrow{01}\right)$ of $G_{\mathbf{Q}\left(\mu_{m}\right)}$.

In Part III we shall need a special case of the equality from Corollary 11.2.2.

COROLlary 11.2.3. Let $\xi_{m}$ be a primitive $m$-th root of 1 . Then we have

$$
m^{n-1}\left(\sum_{k=0}^{m-1} \mathcal{L}_{n}\left(\xi_{m}^{k}\right)\right)=\mathcal{L}_{n}(1)
$$

on the subgroup $H_{n}\left(\mathbf{P}_{\mathbf{Q}\left(\mu_{m}\right)} \backslash\left\{0, \mu_{m}, \infty\right\} ; \overrightarrow{10}, \overrightarrow{01}\right)$ of $G_{\mathbf{Q}\left(\mu_{m}\right)}$, where $\mathcal{L}_{n}(1):=$ $\mathcal{L}_{n}(\overrightarrow{10})$.

Both corollaries follow immediately from Theorem 11.2.1. We give however a detailed proof of Corollary 11.2.3 because of it importance in Part III.

Proof of Corollary 11.2.3. We shall use the notation of Subsection 11.1, where we discussed the $l$-adic analog of the functional equation $L i_{2}\left(z^{m}\right)=$ $m\left(\sum_{i=0}^{m} L i_{2}\left(\xi_{m}^{i} z\right)\right)$. We shall use also the following notation. If $a$ and $b$ are 
elements of a group then $\left(a, b^{1}\right):=(a, b)=a \cdot b \cdot a^{-1} \cdot b^{-1}$ and $\left(a, b^{n}\right):=$ $\left(\left(a, b^{n-1}\right), b\right)$ for $n>1$.

We recall that $Y=\mathbf{P}_{\mathbf{Q}\left(\mu_{m}\right)}^{1} \backslash\left\{0, \mu_{m}, \infty\right\}$ and $f: Y \rightarrow V$ is given by $f(z)=z^{m}$. Let $p$ be a path from $\overrightarrow{01}$ to $\overrightarrow{10}$, the interval [0,1]. Let $\sigma \in H_{n}(Y ; \overrightarrow{10}, \overrightarrow{01})$. Then we have

$$
\mathfrak{f}_{p}(\sigma) \equiv\left(y_{0}, x^{\prime n-1}\right)^{\nu_{0}^{n}(\overrightarrow{10})(\sigma)} \ldots\left(y_{m-1}, x^{\prime n-1}\right)^{\nu_{m-1}^{n}(\overrightarrow{10})(\sigma)}
$$

modulo a subgroup generated by $\Gamma^{n+1} \pi_{1}\left(Y_{\bar{K}} ; \overrightarrow{01}\right)$ and commutators which contain at least two $y$ 's. Observe that $f(p)$ is a path from $\overrightarrow{01}$ to $m \cdot \overrightarrow{10}$. Then for any $\sigma \in H_{n}(V ; \overrightarrow{10}, \overrightarrow{01})$, and therefore also for any $\sigma \in H_{n}(Y ; \overrightarrow{10}, \overrightarrow{01})$ we have

$$
\mathfrak{f}_{f(p)}(\sigma) \equiv\left(y, x^{n-1}\right)^{\kappa_{\overrightarrow{10}}^{n}(\sigma)} \bmod G_{n+1} .
$$

It follows from the equality $f_{*} \mathfrak{f}_{p}=\mathfrak{f}_{f(p)}$ that

$$
m^{n-1}\left(\nu_{0}^{n}(\overrightarrow{10})+\cdots+\nu_{m-1}^{n}(\overrightarrow{10})\right)=\kappa_{\overrightarrow{10}}^{n}
$$

on $H_{n}(Y ; \overrightarrow{10}, \overrightarrow{01})$. We recall that $f_{i}: Y \rightarrow V$ is given by $f_{i}(z)=\xi_{m}^{-i} \cdot z$. Observe that $\left(f_{i}\right)_{*} \mathfrak{f}_{p q_{i}}(\sigma) \equiv\left(y, x^{n-1}\right)^{\nu_{i}(\overrightarrow{10})(\sigma)} \bmod G_{n+1}$ for $\sigma \in H_{n}(Y ; \overrightarrow{10}$, $\left.\overrightarrow{0 \xi_{m}^{i}}\right)=H_{n}(Y ; \overrightarrow{10}, \overrightarrow{01})$ and $\mathfrak{f}_{f_{i}\left(p q_{i}\right)}(\sigma) \equiv\left(y, x^{n-1}\right)^{\kappa_{\xi_{m}^{-i}}^{n}(\sigma)} \bmod G_{n+1}$ for $\sigma \in$ $H_{n}\left(Y ; \overrightarrow{\xi_{n}^{-i} 0}, \overrightarrow{01}\right)=H_{n}(Y ; \overrightarrow{10}, \overrightarrow{01})$, where $q_{i}$ is a path from $\overrightarrow{0 \xi_{m}^{i}}$ to $\overrightarrow{01}$ as on Picture 4. Hence we get

$$
\nu_{i}^{n}(\overrightarrow{10})=\kappa_{\xi_{m}^{-i}}^{n}
$$

on $H_{n}\left(Y ; \overrightarrow{\xi_{n}^{-i} 0}, \overrightarrow{01}\right)=H_{n}(Y ; \overrightarrow{10}, \overrightarrow{01})$. It follows from (11.2.4) and (11.2.5) that

$$
m^{n-1}\left(\sum_{i=0}^{m-1} \kappa_{\xi_{m}^{-i}}^{n}\right)=\kappa_{\overrightarrow{10}}^{n}
$$

on $H_{n}\left(Y ; \overrightarrow{\xi_{n}^{-i} 0}, \overrightarrow{01}\right)=H_{n}(Y ; \overrightarrow{10}, \overrightarrow{01})$. For $\sigma \in H_{n}(Y ; \overrightarrow{10}, \overrightarrow{01})$ we have $\kappa_{\xi_{m}^{-i}}^{n}(\sigma)=\mathcal{L}_{n}\left(\xi_{m}^{-i}\right)(\sigma)$ and $\kappa_{\overrightarrow{10}}^{n}(\sigma)=\mathcal{L}_{n}(\overrightarrow{10})(\sigma)$. This finishes the proof of Corollary 11.2.3.

One of the most useful functional equations of classical polylogarithms is the relation between $L i_{n}(z)$ and $L i_{n}\left(\frac{1}{z}\right)$. For $l$-adic polylogarithms we have the following result. 
Corollary 11.2.6. For any $z \in V(K)$, we have

$$
\mathcal{L}_{n}(z)+(-1)^{n} \mathcal{L}_{n}\left(\frac{1}{z}\right)=0
$$

on the subgroup $H_{n}\left(V_{\overline{\mathbf{Q}}} ; z, \overrightarrow{01}\right)$.

Proof. It follows from Theorem 11.2.1 that

$$
\mathcal{L}_{n}(z)-\mathcal{L}_{n}(\overrightarrow{01})+(-1)^{n}\left(\mathcal{L}_{n}\left(\frac{1}{z}\right)-\mathcal{L}_{n}(\overrightarrow{\infty 1})\right)=0
$$

$\mathcal{L}_{n}(\overrightarrow{01})$ vanishes. Hence we have to calculate $\mathcal{L}_{n}(\overrightarrow{\infty 1})$. Let $p$ a path from $\overrightarrow{01}$ to $\overrightarrow{10}$ and let $s$ a path from $\overrightarrow{10}$ to $\overrightarrow{1 \infty}$ as on the picture.

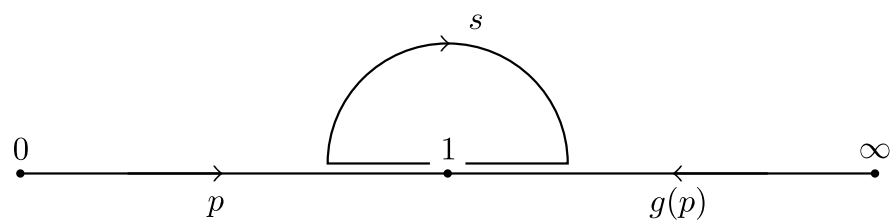

Picture 10

Let $g: V \rightarrow V$ be given by $g(z)=\frac{1}{z}$. Let us set $q:=g(p)^{-1} \cdot s \cdot p$. We denote by $\pi^{\prime \prime}$ the subgroup $\left[\Gamma^{2} \pi_{1}\left(V_{\bar{K}} ; \overrightarrow{01}\right), \Gamma^{2} \pi_{1}\left(V_{\bar{K}} ; \overrightarrow{01}\right)\right]$ of $\pi_{1}\left(V_{\bar{K}} ; \overrightarrow{01}\right)$. Let $\left(\Gamma^{n+1} \pi_{1}\left(V_{\bar{K}} ; \overrightarrow{01}\right), \pi^{\prime \prime}\right)$ be a normal subgroup of $\pi_{1}\left(V_{\bar{K}} ; \overrightarrow{01}\right)$ generated by $\Gamma^{n+1} \pi_{1}\left(V_{\bar{K}} ; \overrightarrow{01}\right)$ and $\pi^{\prime \prime}$.

Let $\sigma \in H_{n}\left(V_{\overline{\mathbf{Q}}} ; z, \overrightarrow{01}\right)$. Then we have

$$
\begin{aligned}
\mathfrak{f}_{q}(\sigma)= & \left.\prod_{i+j=n, i \geq 1, j \geq 1}\left((y, x) x^{i-1}\right) y^{j-1}\right)^{\kappa_{i, j}(\overrightarrow{\infty 1})(\sigma)} \\
& \bmod \left(\Gamma^{n+1} \pi_{1}\left(V_{\bar{K}}, \overrightarrow{01}\right), \pi^{\prime \prime}\right) .
\end{aligned}
$$

for some $\kappa_{i, j}(\overrightarrow{\infty 1})(\sigma) \in \mathbf{Z}_{l}$. It follows from Lemma 1.0.6 and from equality (10.0.1) that

$$
\mathfrak{f}_{q}=q^{-1} \cdot g_{*}\left(\mathfrak{f}_{p}\right)^{-1} \cdot q \cdot p^{-1} \cdot \mathfrak{f}_{s} \cdot p \cdot \mathfrak{f}_{p}
$$

Observe that

$$
q^{-1} \cdot g_{*}(y) \cdot q=y \quad \text { and } \quad q^{-1} \cdot g_{*}(x) \cdot q=x^{-1} \cdot y^{-1}
$$


Let $\sigma \in H_{n}\left(V_{\overline{\mathbf{Q}}} ; z, \overrightarrow{01}\right)$. Then we have

$$
\begin{aligned}
\mathfrak{f}_{p}(\sigma)= & \left.\prod_{i+j=n, i \geq 1, j \geq 1}\left((y, x) x^{i-1}\right) y^{j-1}\right)^{\kappa_{i, j}(\overrightarrow{10})(\sigma)} \\
& \bmod \left(\Gamma^{n+1} \pi_{1}\left(V_{\bar{K}}, \overrightarrow{01}\right), \pi^{\prime \prime}\right)
\end{aligned}
$$

for some $\kappa_{i, j}(\overrightarrow{10})(\sigma) \in \mathbf{Z}_{l}$. It follows from (11.2.7)-(11.2.10) that

$$
\kappa_{n-1,1}(\overrightarrow{\infty 1})=(-1)^{n} \kappa_{n-1,1}(\overrightarrow{10})(\sigma)+\kappa_{n-1,1}(\overrightarrow{10})(\sigma)
$$

Hence $\kappa_{n-1,1}(\overrightarrow{\infty 1})=0$ if $n$ is odd.

We shall show that $\kappa_{n-1,1}(\overrightarrow{10})$ vanishes for $n$ even. Let $x, y$ and $z$ be generators of $\pi_{1}\left(V_{\bar{K}} ; \overrightarrow{01}\right)$ as on the picture.

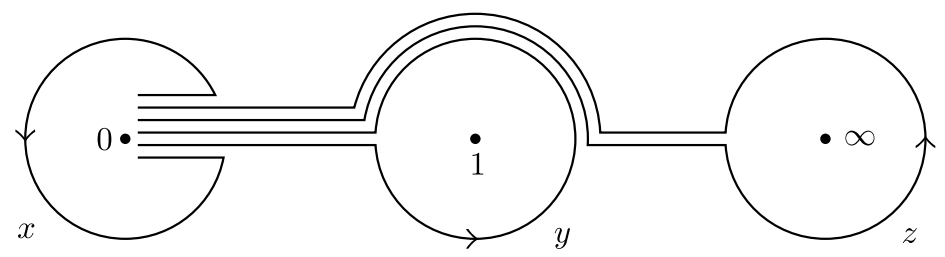

Picture 11

Then we have $z \cdot y \cdot x=1$. It follows from Proposition 2.2.1 that

$$
\begin{aligned}
\left(\mathfrak{f}_{q}(\sigma)(x, y)\right)^{-1} \cdot z^{\chi(\sigma)} \cdot\left(\mathfrak{f}_{q}(\sigma)(x, y)\right) \cdot\left(\mathfrak{f}_{p}(\sigma)(x, y)\right)^{-1} \cdot y^{\chi(\sigma)} & \cdot\left(\mathfrak{f}_{p}(\sigma)(x, y)\right) \cdot x^{\chi(\sigma)}=1 .
\end{aligned}
$$

Let $\sigma \in H_{n}\left(V_{\bar{K}} ; z, \overrightarrow{01}\right)$. It follows from (11.2.8) and (11.2.9) that

$$
\mathfrak{f}_{q}(\sigma)(x, y)=\left(\mathfrak{f}_{p}(\sigma)\left(x^{-1} y^{-1}, y\right)\right)^{-1} \cdot\left(\mathfrak{f}_{p}(\sigma)(x, y)\right) .
$$

Hence we get

$$
\begin{gathered}
\left(\mathfrak{f}_{p}(\sigma)(x, y)\right)^{-1} \cdot\left(\mathfrak{f}_{p}(\sigma)\left(x^{-1} y^{-1}, y\right)\right) \cdot x^{-1} \cdot y^{-1} \cdot\left(\mathfrak{f}_{p}(\sigma)\left(x^{-1} y^{-1}, y\right)\right)^{-1} \\
\cdot\left(\mathfrak{f}_{p}(\sigma)(x, y)\right) \cdot\left(\mathfrak{f}_{p}(\sigma)(x, y)\right)^{-1} \cdot y \cdot\left(\mathfrak{f}_{p}(\sigma)(x, y)\right) \cdot x=1 .
\end{gathered}
$$

Comparing exponents at $\left(y, x^{n}\right)$ we get $\left(1+(-1)^{n}\right) \kappa_{n-1,1}(\overrightarrow{10})=0$. Hence $\kappa_{n-1,1}(\overrightarrow{10})=0$ for $n$ even (see also [I1], [I2] and [D], where the element $\mathfrak{f}_{p}(\sigma)$ is studied). Therefore $\kappa_{n-1,1}(\overrightarrow{\infty 1})=0$ for any $n$. The equality $\kappa_{n-1,1}(\overrightarrow{\infty 1})=\mathcal{L}_{n}(\overrightarrow{\infty 1})$ implies the corollary. 
The fact that $\kappa_{n-1,1}(\overrightarrow{10})$ vanishes for $n$ even implies the following well known result.

Corollary 11.2.11.

$$
\mathcal{L}_{2 n}(\overrightarrow{10})=0
$$

\section{$\S 12$. Monodromy of $l$-adic iterated integrals and $l$-adic polyloga- rithms}

12.0. We shall show here that suitable defined $l$-adic polylogarithms form a local system with the similar shape of the monodromy representation as the local system of classical polylogarithms given in [BD]. We start with the discussion of the monodromy of arbitrary $l$-adic iterated integrals. The notation is the same as in Section 10.

Let $p$ be a path from $v$ to $z$ on $X_{\bar{K}}$ and let $S \in \pi_{1}\left(X_{\bar{K}} ; v\right)$. Then we have

$$
\mathfrak{f}_{p S}(\sigma)=S^{-1} \cdot \mathfrak{f}_{p}(\sigma) \cdot S \cdot \mathfrak{f}_{S}(\sigma) \text {. }
$$

Let $\operatorname{Map}\left(G_{K} ; \pi_{1}\left(X_{\bar{K}} ; v\right)\right)$ be the set of all maps from $G_{K}$ to $\pi_{1}\left(X_{\bar{K}} ; v\right)$. We define a map

$$
r_{z, v ; p}: \pi_{1}\left(X_{\bar{K}} ; v\right) \longrightarrow \operatorname{Aut}_{s e t}\left(\operatorname{Map}\left(G_{K} ; \pi_{1}\left(X_{\bar{K}} ; v\right)\right)\right)
$$

setting

$$
r_{z, v ; p}(S)(w)(\sigma):=S^{-1} \cdot w(\sigma) \cdot S \cdot \mathfrak{f}_{S}(\sigma),
$$

for $S \in \pi_{1}\left(X_{\bar{K}} ; v\right), w \in \operatorname{Map}\left(G_{K} ; \pi_{1}\left(X_{\bar{K}} ; v\right)\right)$ and $\sigma \in G_{K}$.

Further we drop the indices $z, v ; p$ to simplify the notation.

Lemma 12.0.1. The map $r_{z, v ; p}$ is a representation of $\pi_{1}\left(X_{\bar{K}} ; v\right)$.

Proof. Let $S, T \in \pi_{1}\left(X_{\bar{K}} ; v\right)$. We have $r(T)(r(S) w)(\sigma)=T^{-1}\left(S^{-1}\right.$. $\left.w(\sigma) \cdot S \cdot \mathfrak{f}_{S}(\sigma)\right) \cdot T \cdot \mathfrak{f}_{T}(\sigma)=(S \cdot T)^{-1} \cdot w(\sigma) \cdot(S \cdot T) \cdot\left(T^{-1} \cdot \mathfrak{f}_{S}(\sigma) \cdot T \cdot \mathfrak{f}_{T}(\sigma)\right)=$ $(S \cdot T)^{-1} \cdot w(\sigma) \cdot(S \cdot T) \cdot \mathfrak{f}_{S T}(\sigma)=r(S \cdot T)(w)(\sigma)$. We recall that in our notation $S \cdot T$ means that first we go along $T$ and then along $S$. Therefore $r$ is a representation of $\pi_{1}\left(X_{\bar{K}} ; v\right)$.

We recall that $k_{x}: \pi_{1}\left(X_{\bar{K}} ; v\right) \rightarrow \mathbf{Q}_{l}\{\{\mathbf{X}\}\}$ is a continous multiplicative embedding given by $k_{x}\left(x_{i}\right)=e^{X_{i}}$ for $i=1, \ldots, n$ and that for a path $p$ from $v$ to $z$ we set $\Lambda_{p}(\sigma):=k_{x}\left(\mathfrak{f}_{p}(\sigma)\right)$. 
Let $\operatorname{Map}\left(G_{K} ; \mathbf{Q}_{l}\{\{\mathbf{X}\}\}\right)$ be the set of all maps from $G_{K}$ to $\mathbf{Q}_{l}\{\{\mathbf{X}\}\}$. Observe that $\operatorname{Map}\left(G_{K} ; \mathbf{Q}_{l}\{\{\mathbf{X}\}\}\right)$ is a vector space over $\mathbf{Q}_{l}$. We denote by $G L\left(\operatorname{Map}\left(G_{K} ; \mathbf{Q}_{l}\{\{\mathbf{X}\}\}\right)\right)$ the group of linear automorphisms of the vector space $\operatorname{Map}\left(G_{K} ; \mathbf{Q}_{l}\{\{\mathbf{X}\}\}\right)$.

Let us define a map

$$
R_{z, v ; p}: \pi_{1}\left(X_{\bar{K}} ; v\right) \longrightarrow G L\left(\operatorname{Map}\left(G_{K} ; \mathbf{Q}_{l}\{\{\mathbf{X}\}\}\right)\right)
$$

setting

$$
R_{z, v ; p}(S)(W)(\sigma):=k_{x}(S)^{-1} \cdot W(\sigma) \cdot k_{x}(S) \cdot \Lambda_{S}(\sigma)
$$

Proposition 12.0.2. The map $R_{z, v ; p}$ is a representation of $\pi_{1}\left(X_{\bar{K}} ; v\right)$.

Proof. To simplify the notation let us set $R=R_{z, v ; p}$. Let $S, T \in$ $\pi_{1}\left(X_{\bar{K}} ; v\right)$. We have $R(T)(R(S)(W))(\sigma)=k_{x}(T)^{-1} \cdot(R(S)(W)(\sigma)) \cdot k_{x}(T)$. $\Lambda_{T}(\sigma)=k_{x}(T)^{-1} \cdot\left(k_{x}(S)^{-1} \cdot W(\sigma) \cdot k_{x}(S) \cdot \Lambda_{S}(\sigma)\right) \cdot k_{x}(T) \cdot \Lambda_{T}(\sigma)=k_{x}(S \cdot$ $T)^{-1} \cdot W(\sigma) \cdot k_{x}(S \cdot T) \cdot k_{x}(T)^{-1} \cdot \Lambda_{S}(\sigma) \cdot k_{x}(T) \cdot \Lambda_{T}(\sigma)=R(S \cdot T)(W)(\sigma)$.

It follows from Lemma 10.3.1 that the embedding $k_{x}: \pi_{1}\left(X_{\bar{K}} ; v\right) \rightarrow$ $\mathbf{Q}_{l}\{\{\mathbf{X}\}\}$ extends uniquely to a continous multiplicative embedding $\bar{k}_{x}$ : $\pi_{1}\left(X_{\bar{K}} ; v\right) \otimes \mathbf{Q} \rightarrow \mathbf{Q}_{l}\{\{\mathbf{X}\}\}$.

Proposition 12.0.3. The representation $R_{z, v ; p}$ extends to the representation

$$
\bar{R}_{z, v ; p}: \pi_{1}\left(X_{\bar{K}} ; v\right) \otimes \mathbf{Q} \longrightarrow G L\left(\operatorname{Map}\left(G_{K} ; \mathbf{Q}_{l}\{\{\mathbf{X}\}\}\right)\right)
$$

Let $S \in \pi_{1}\left(X_{\bar{K}} ; v\right) \otimes \mathbf{Q}$. Then we have

$$
\bar{R}_{z, v ; p}(S)(W)(\sigma)=\bar{k}_{x}(S)^{-1} \cdot W(\sigma) \cdot \bar{k}_{x}(S) \cdot \bar{R}_{z, v ; p}(S)(1)(\sigma) .
$$

Proof. We define an increasing filtration $\left\{\mathcal{W}_{-i}\right\}_{i \in \mathbf{N}}$ of the $\mathbf{Q}_{l}$-vector space $\operatorname{Map}\left(G_{K} ; \mathbf{Q}_{l}\{\{\mathbf{X}\}\}\right)$ setting

$$
\mathcal{W}_{-2 k}=\mathcal{W}_{-2 k-1} \text { to be a set of all maps from } G_{K} \text { to } I^{k}
$$

where $I^{k}$ is a $k$-th power of the augmentation ideal of $\mathbf{Q}_{l}\{\{\mathbf{X}\}\}$. Let $S \in$ $\pi_{1}\left(X_{\bar{K}} ; v\right)$ and let $W \in \mathcal{W}_{-2 k}$. Then we have

$$
R_{z, v ; p}(S)(W) \equiv W \bmod \mathcal{W}_{-2(k+1)}
$$


Hence the image of $R_{z, v ; p}$ is in the subgroup of pro-unipotent automorphisms of the vector space $\operatorname{Map}\left(G_{K} ; \mathbf{Q}_{l}\{\{\mathbf{X}\}\}\right)$. This implies that the representation $R_{z, v ; p}$ extends to the representation

$$
\bar{R}_{z, v ; p}: \pi_{1}\left(X_{\bar{K}} ; v\right) \otimes \mathbf{Q} \rightarrow G L\left(\operatorname{Map}\left(G_{K} ; \mathbf{Q}_{l}\{\{\mathbf{X}\}\}\right)\right) .
$$

Let $S \in \pi_{1}\left(X_{\bar{K}} ; v\right) \otimes \mathbf{Q}$ be such that $S^{l^{m}} \in \pi_{1}\left(X_{\bar{K}} ; v\right)$. Then we have

$$
R_{z, v ; p}\left(S^{l^{m}}\right)(W)(\sigma)=k_{x}\left(S^{l^{m}}\right)^{-1} \cdot W(\sigma) \cdot k_{x}\left(S^{l^{m}}\right) \cdot \Lambda_{S^{l m}}(\sigma),
$$

where $\Lambda_{S^{l}}(\sigma)=R_{z, v ; p}\left(S^{l^{m}}\right)(1)(\sigma)$. This implies that

$$
\bar{R}_{z, v ; p}(S)(W)(\sigma)=\bar{k}_{x}(S)^{-1} \cdot W(\sigma) \cdot \bar{k}_{x}(S) \cdot \bar{R}_{z, v ; p}(S)(1)(\sigma) .
$$

The elements $S \in \pi_{1}\left(X_{\bar{K}} ; v\right) \otimes \mathbf{Q}$ such that $S^{l^{m}} \in \pi_{1}\left(X_{\bar{K}} ; v\right)$ for some $m$ are dense in $\pi_{1}\left(X_{\bar{K}} ; v\right) \otimes \mathbf{Q}$ hence the last formula holds for any $S \in$ $\pi_{1}\left(X_{\bar{K}} ; v\right) \otimes \mathbf{Q}$. This finishes the proof of the proposition.

12.1. Now we shall study monodromy of $l$-adic polylogarithms, more exactly, we shall study monodromy of coefficients at $X^{n-1} Y$ of the power series $\Lambda_{p}(\sigma)$. Let $V=\mathbf{P}_{K}^{1} \backslash\{0,1, \infty\}$ and let $p$ be a path from $\overrightarrow{01}$ to $z$. From now on the notation is the same as in Subsection 11.0.

We define functions $\lambda_{i}(z)_{p}, \mu_{j}(z)_{p}$ and $\nu_{i, j}(z)_{p}$ from $G_{K}$ to $\mathbf{Q}_{l}$ by the congruence

$$
\begin{aligned}
\Lambda_{p}(\sigma) \equiv 1 & +\sum_{k=1}^{\infty} \frac{\left(l(z)_{p}(\sigma)\right)^{k}}{k !} X^{k}+\sum_{i=1}^{\infty} \lambda_{i}(z)_{p}(\sigma) X^{i-1} Y \\
& +\sum_{j=2}^{\infty} \mu_{j}(z)_{p}(\sigma) Y X^{j-1}+\sum_{i, j=1}^{\infty} \nu_{i, j}(z)_{p}(\sigma) X^{i} Y X^{j}
\end{aligned}
$$

modulo the ideal generated by monomials with at least two $Y$ 's.

The function $\lambda_{1}(z)_{p}=l_{1}(z)_{p}$ and the $l$-adic polylogarithms $l_{k}(z)_{p}$ can be expressed by the function $\lambda_{k}(z)_{p}$ and the functions $l(z)_{p}$ and $\lambda_{i}(z)_{p}$ with $i<k$.

Proposition 12.1.1. The monodromy transformation of functions $l(z)_{p}$ and $\lambda_{n}(z)_{p}$ is as follows:

$$
\begin{gathered}
x: l(z)_{p} \longrightarrow l(z)_{p}+(\chi-1), \quad \lambda_{n}(z)_{p} \longrightarrow \lambda_{n}(z)_{p}+\sum_{i=1}^{n-1} \frac{(-1)^{n-i}}{(n-i) !} \lambda_{i}(z)_{p}, \\
\mu_{n}(z)_{p} \longrightarrow \mu_{n}(z)_{p}+\sum_{i=2}^{n-1} \frac{\chi^{n-i}}{(n-i) !} \mu_{i}(z)_{p}+\frac{\chi^{n-1}}{(n-1) !} \lambda_{1}(z)_{p}
\end{gathered}
$$


and

$$
\begin{gathered}
y: l(z)_{p} \longrightarrow l(z)_{p}, \quad \lambda_{1}(z)_{p} \longrightarrow \lambda_{1}(z)_{p}+(\chi-1), \\
\lambda_{n}(z)_{p} \longrightarrow \lambda_{n}(z)_{p}+\chi \frac{\left(l(z)_{p}\right)^{n-1}}{(n-1) !} \\
\text { for } n>1 \text { and } \mu_{n}(z)_{p} \rightarrow \mu_{n}(z)_{p}-\frac{\left(l(z)_{p}\right)^{n-1}}{(n-1) !}
\end{gathered}
$$

Proof. The proposition follows from the formula

$$
\Lambda_{p \cdot S}(\sigma)=k(S)^{-1} \cdot \Lambda_{p}(\sigma) \cdot k(S) \cdot \Lambda_{S}(\sigma)
$$

which for $S=x$ gives

$$
\Lambda_{p \cdot x}(\sigma)=e^{-X} \cdot \Lambda_{p}(\sigma) \cdot e^{\chi(\sigma) X}
$$

For $S=y$ the formula is more complicated, however when we restrict our attention to coefficients with only one $Y$ then the formula have the same simple form.

\section{REFERENCES}

[B] N. Bourbaki, Eléments de mathématiques, Groupes et algèbres de Lie, Diffusion C.C.L.S., Paris, 1972.

[BD] A. A. Beilinson and P. Deligne, Interprétation motivique de la conjecture de Zagier reliant polylogarithmes et régulareurs, Motives (U. Jannsen, S. L. Kleiman and J.-P. Serre, eds.), Proc. of Symp. in Pure Math. 55, Part II, AMS (1994), pp. $97-121$.

[Ch] K. T. Chen, Iterated integrals, fundamental groups and covering spaces, Trans. of the Amer. Math. Soc., 206 (1975), 83-98.

[D] P. Deligne, Le groupe fondamental de la droite projective moins trois points, Galois Groups over Q (Y. Ihara, K. Ribet and J.-P. Serre, eds.), Mathematical Sciences Research Institute Publications, no 16 (1989), pp. 79-297.

[I1] Y. Ihara, Profinite braid groups, Galois representations and complex multiplications, Annals of Math., 123 (1986), 43-106.

[I2] Y. Ihara, Braids, Galois Groups and Some Arithmetic Functions, Proc. of the Int. Cong. of Math. Kyoto (1990), 99-119.

[W1] Z. Wojtkowiak, The Basic Structure of Polylogarithmic Functional Equations, Structural Properties of Polylogarithms (L. Lewin, ed.), Mathematical Surveys and Monographs, Vol 37, pp. 205-231. 
Université de Nice-Sophia Antipolis

Département de Mathématiques

Laboratoire Jean Alexandre Dieudonné

U.R.A. au C.N.R.S., No 168

Parc Valrose - B.P.N $N^{\circ} 71$

06108 Nice Cedex 2

France

wojtkow@math.unice.fr 